\title{
Liouville Correlation Functions from Four-dimensional Gauge Theories
}

\author{
Luis F. Alday, Davide Gaiotto and Yuji Tachikawa \\ School of Natural Sciences, Institute for Advanced Study, \\ Princeton, NJ 08540, USA \\ alday, dgaiotto, yujitach@ias.edu
}

\begin{abstract}
We conjecture an expression for the Liouville theory conformal blocks and correlation functions on a Riemann surface of genus $g$ and $n$ punctures as the Nekrasov partition function of a certain class of $\mathcal{N}=2$ SCFTs recently defined by one of the authors. We conduct extensive tests of the conjecture at genus 0,1 .
\end{abstract}

Keywords: Conformal field theory, gauge theory

MSC: $81 \mathrm{~T} 40,81 \mathrm{~T} 60$ 


\section{Contents}

1. Introduction 2

2. Review: a class of four dimensional $\mathcal{N}=2$ SCFTs 3

3. Instanton sums and the conformal blocks 6

3.1 Nekrasov's partition function 7

3.2 Sphere with four punctures 8

3.3 Torus with one puncture 10

3.4 Sphere with multiple punctures 10

3.5 Torus with multiple punctures 11

4. Liouville correlators 11

4.1 Sphere with four punctures 12

4.2 Torus with one puncture 13

4.3 General proposal 14

5. Seiberg-Witten differential and the insertion of $T(z)$

6. Conclusions and Open Problems 16

A. Liouville theory 19

A.1 Conformal blocks 19

A.2 The DOZZ formula 22

B. Nekrasov formulae 23

B.1 Instanton part 23

B.2 One-loop part 25

B.3 Example 27

C. $U(1)$ factors 28

C.1 Sphere 28

C.2 Torus 29 


\section{Introduction}

In [1] it was shown that a large class of $\mathcal{N}=2$ superconformal field theories (SCFTs) in four dimensions arise by compactifying the six-dimensional $(2,0)$ theory of type $A_{1}$ on a Riemann surface with punctures. A canonical example is $\mathcal{N}=4 S U(2)$ gauge theory, which is the compactification of this $6 \mathrm{~d}$ theory on a torus whose modulus is the marginal gauge coupling parameter $\tau$. The $S L(2, \mathbb{Z})$ S-duality of $\mathcal{N}=4$ theory is geometrically realized as the modular transformation of the torus.

Each SCFT in the class is thus labeled by two integers $g, n$, which are the genus and the number of punctures of the Riemann surface. The parameter space of the theory coincides with the complex structure moduli space of the punctured Riemann surface. Each distinct way to sew such Riemann surface together from pairs of pants corresponds to a different Lagrangian description of the theory, and a Lagrangian description is weakly coupled in the region of parameter space where the pairs of pants are sewn together by long tubes. Each tube represents an $S U(2)$ gauge group. Each pair of pants represents a "block" of matter hypermultiplets. The sewing of the Riemann surface encodes the detailed structure of the matter representations.

It was also shown in [1] that each puncture is associated to an $S U(2)$ flavor symmetry, which can be used to give mass to the hypermultiplets. We have already mentioned that the compactification on a torus without a puncture gives rise to the $\mathcal{N}=4$ theory. Other basic examples are a sphere with four punctures which gives $\mathcal{N}=2 S U(2)$ theory with $N_{f}=4$ flavors, and a torus with one puncture which gives $\mathcal{N}=2^{*} S U(2)$ theory, i.e. $\mathcal{N}=4$ theory deformed by the mass to the adjoint hypermultiplet. The former has $S O(8)$ flavor symmetry which contains as a subgroup $S U(2)^{4}$, each $S U(2)$ factor corresponding to each of the punctures; the mass parameter of the latter is associated to the $S U(2)$ flavor symmetry acting on the adjoint hypermultiplet. Thus each puncture is associated with a number, which is the mass parameter of the $S U(2)$ flavor symmetry associated to it.

Basic operations which are familiar from the theory of sewing Riemann surfaces have a direct translation in the language of these $\mathcal{N}=2$ field theories. The basic operations which relate different sewings of the same Riemann surface are elementary S-duality transformations, which relate different Lagrangian descriptions of the same theory. Sewing two Riemann surfaces together, or adding a handle to a Riemann surface also map to very natural operations on the corresponding $\mathcal{N}=2$ SCFTs: they correspond to gauging the diagonal subgroup of two $S U(2)$ flavor symmetries at the two punctures sewed together.

There is a huge physical and mathematical literature on $\mathcal{N}=2$ field theories. It is natural to wonder if this class of SCFTs may provide a connection with the large literature on objects defined through the sewing of Riemann surfaces, in particular the theory of $2 \mathrm{~d}$ conformal field theories. This paper is devoted to test a specific

realization of this general idea: the identification of the Nekrasov partition function 
[2, 3] of these $\mathcal{N}=2$ SCFTs and the Liouville theory correlation functions on the corresponding Riemann surfaces.

The crucial idea is that for each sewing of the Riemann surface one is given two natural objects: Nekrasov's instanton computation in the corresponding Lagrangian description of the theory and the "Liouville conformal block" defined by a sum over Virasoro descendants of a primary field in each of the sewing channels. With a judicious identification of the parameters on the two sides, we will demonstrate by explicit examples that the two objects coincide at genus $g=0,1$ for various $n$. We will also conjecture the general map at higher genus and number of punctures.

Taking inspiration from Pestun's computation [i] of the $S^{4}$ partition function of $\mathcal{N}=2$ SCFTs we will assemble together the squared modulus of Nekrasov's instanton partition function together with tree level and one-loop contributions to produce an S-duality invariant object, which coincides with the Liouville correlation function on the corresponding punctured Riemann surface. We will see that the product of the Liouville three-point functions [5, 6, 7] neatly recombines into the modulus squared of the one-loop contribution to Nekrasov's partition function.

The structure of the paper is the following. We begin in Sec. 2 by reviewing the class of four-dimensional $\mathcal{N}=2$ SCFTs associated to punctured Riemann surfaces. Then in Sec. 3 we formulate the equivalence of Nekrasov's instanton sum associated to this class of theories and the Liouville conformal blocks. In Sec. 专 we go on to show that the Liouville correlators on a sphere or on a torus can be written as an integral of the absolute value squared of Nekrasov's full partition function, including the classical and the one-loop part in addition to the instanton part. In Sec. 5 we briefly discuss how the Seiberg-Witten curve can be recovered from the point of view of the Liouville theory; it involves the insertion of the energy momentum tensor of the $2 \mathrm{~d}$ CFT. As a byproduct of this analysis we are led to a proposal for the quantum version of the Seiberg-Witten curve. We conclude with the discussions of future directions in Sec. 6. There are three appendices: App. A and App. B collect rudimentary facts about the Liouville theory and Nekrasov's instanton counting, respectively. In App. $\mathrm{O}$ we propose how to decouple the $U(1)$ part from Nekrasov's instanton sum of $U(2)$ quiver theories.

\section{Review: a class of four dimensional $\mathcal{N}=2$ SCFTs}

We will denote as $\mathcal{T}_{g, n}$ the four-dimensional SCFT we obtain by compactifying sixdimensional $(2,0)$ theory of type $A_{1}$ on a genus- $g$ Riemann surface with $n$ punctures. The parameter space of gauge couplings is the moduli $\mathcal{M}_{g, n}$ of the genus- $g$ $n$-punctured Riemann surfaces. The surface itself is denoted by $C_{g, n}$, which is sewn from $2 g-2+n$ pairs of pants, joined by $3 g-3+n$ tubes.

The simplest example is the theory associated to a three punctured-sphere, $\mathcal{T}_{0,3}$. $\mathcal{M}_{0,3}$ is a point, and $\mathcal{T}_{0,3}$ is simply a theory of four free hypermultiplets. Four free 
hypermultiplets can be represented by eight $\mathcal{N}=1$ free chiral multiplets transforming in $\mathbf{2}_{a} \otimes \mathbf{2}_{b} \otimes \mathbf{2}_{c}$ of the flavor symmetry $S U(2)_{a} \times S U(2)_{b} \times S U(2)_{c}$ which commutes with $\mathcal{N}=2$ supercharges. Let us denote the mass parameters associated to the three $S U(2)$ flavor symmetries as $m_{a}, m_{b}$ and $m_{c}$ respectively. Then the masses of the hypermultiplets are

$$
m_{a} \pm m_{b} \pm m_{c} .
$$

When sewing pairs of pants together, one always gauges a diagonal combination of two such $S U(2)$ flavor symmetry groups. If the groups belong to different pairs of pants, the $S U(2)$ gauge group is coupled to a total of 8 hypermultiplets, i.e. four fundamental $S U(2)$ matter representations. If the two flavor groups belong to the same set of hypermultiplets, they represent an adjoint plus a singlet of $S U(2)$. All in all, at the end we are left with $n$ residual $S U(2)$ flavor symmetry groups, each associated to a puncture of $C_{g, n}$. In the following we will label the flavor groups as $S U(2)_{a, b, c, \ldots}$ and the corresponding mass parameters as $m_{a, b, c, \cdots}^{2}$. We will also denote the $S U(2)$ gauge groups as $S U(2)_{1,2,3, \ldots}$. We denote the Coulomb branch order parameters as $a_{i=1,2,3, \cdots}$. Semi-classically they are the diagonal components of the adjoint scalar, $\phi_{i}=\operatorname{diag}\left(a_{i},-a_{i}\right)$. More precisely they are special coordinates controlling the mass of the BPS particles. If we keep track of the flavor symmetry groups, the natural gauge coupling parameter space is the moduli space of a Riemann surface with $n$ distinct punctures.

The next simplest example is the $\mathcal{T}_{0,4}$ theory. A sphere with four punctures can be assembled from two pairs of pants joined by a tube. All weakly coupled realizations of the theory involve a single $S U(2)$ gauge group, coupled to a total of four fundamental hypermultiplets: $S U(2) N_{f}=4$. This theory is very well studied [8]. It has an overall $S O(8)$ flavor symmetry group, a marginal gauge coupling $\tau$ and a peculiar S-duality group: it is $S L(2, \mathbb{Z})$, but some S-duality transformations exchange the standard matter fields in the $\boldsymbol{8}_{v}$ representation of $S O(8)$ with new matter fields in $\boldsymbol{8}_{s}$ or $\boldsymbol{8}_{c}$ representation.

From the point of view of the pants decomposition, it is natural to consider the two groups of two fundamental hypermultiplets separately, and focus on an $S O(4) \times$ $S O(4) \sim S U(2)_{a} \times S U(2)_{b} \times S U(2)_{c} \times S U(2)_{d}$ subgroup of the flavor group. Then

$$
\begin{aligned}
& \mathbf{8}_{v} \sim\left(\mathbf{2}_{a} \otimes \mathbf{2}_{b}\right) \oplus\left(\mathbf{2}_{c} \otimes \mathbf{2}_{d}\right), \\
& \mathbf{8}_{s} \sim\left(\mathbf{2}_{a} \otimes \mathbf{2}_{c}\right) \oplus\left(\mathbf{2}_{b} \otimes \mathbf{2}_{d}\right), \\
& \mathbf{8}_{c} \sim\left(\mathbf{2}_{a} \otimes \mathbf{2}_{d}\right) \oplus\left(\mathbf{2}_{c} \otimes \mathbf{2}_{b}\right) .
\end{aligned}
$$

We recognize the three possible ways to decompose the four-punctured sphere in two pairs of pants, distributing the four punctures $a, b, c, d$ in various ways among the pants. They correspond to distinct weakly-coupled limits of the same theory, see Figure 1. Let $m_{a}$ be the mass parameter associated to $S U(2)_{a}$, etc. Then the mass 

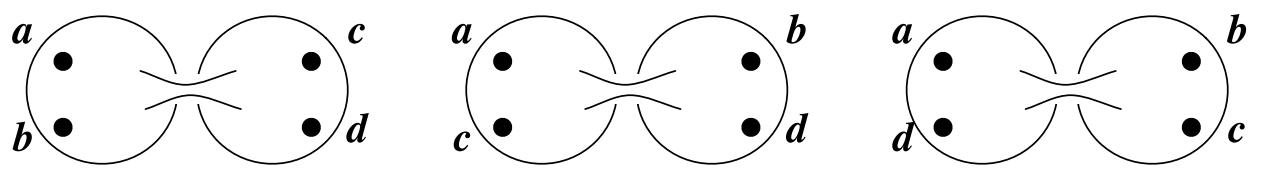

Figure 1: Three distinct ways to decompose a four-punctured sphere into two pairs of pants. They correspond to three distinct weakly-coupled frames of $S U(2)$ gauge theory with four flavors.

eigenvalues of the four hypermultiplets in $\mathbf{8}_{v}$ is

$$
m_{a} \pm m_{b}, \quad m_{c} \pm m_{d} .
$$

There are two natural ways to parameterize the modulus of the sphere with four punctures. One is to take the cross ratio $q$ of the four punctures. $q$ lives in $\mathbb{C P}^{1} \backslash\{0,1, \infty\}$. The other is to take the double cover of the sphere and to take the modulus $\tau_{\text {IR }}$ of the resulting torus, which is the Seiberg-Witten curve when all masses are set to zero. $\tau_{\text {IR }}$ parameterizes an upper half plane which can be seen as the universal cover of the punctured sphere parameterized by $q$.

In the early literature on the subject 8$] \tau_{\mathrm{IR}}$ was identified with $\tau_{\mathrm{UV}}$. This proposal was invalidated by explicit instanton computations [9]. As we will review in Appendix B.3, the cross ratio is actually given by the exponential of the UV coupling, $q=\exp \left(2 \pi i \tau_{\mathrm{UV}}\right)$; this precise relation was first noticed by 10 .

Note that $q$ is also the sewing parameter for the four-punctured sphere. This result is much more natural in our general setup: the universal cover of the space of the marginal couplings, that is the moduli space $\mathcal{M}_{g, n}$ of $n$ punctures on a genus- $g$ Riemann surface in general is very intricate, and bears no obvious resemblance to the product of upper half planes parameterized by the gauge couplings $\tau_{i}$ of the $S U(2)_{i}$ gauge groups. It is also distinct from the space of IR gauge couplings, even for zero masses, which actually depend on the Coulomb branch parameters as well. On the other hand the set of sewing parameters $q_{i}$ could be easily matched to the UV gauge couplings as $q_{i}=\exp \left(2 \pi i \tau_{i, \mathrm{UV}}\right)$.

Another simple example is $\mathcal{T}_{1,1}$, i.e. a torus with one puncture. It can be assembled from a pair of pants by gluing together two legs. Hence $\mathcal{T}_{1,1}$ coincides with an $S U(2)$ gauge theory with an adjoint hypermultiplet (and one extra free hypermultiplet), i.e. $\mathcal{N}=2^{*} S U(2)$ gauge theory. The gauge coupling $\tau$ naturally parameterizes the complex structure of the torus, and the sewing parameter is indeed $q=\exp (2 \pi i \tau)$ again. In this case there is no distinction between the UV and the IR couplings when the adjoint mass is zero, because the theory is then $\mathcal{N}=4$.

Next consider a more complex example: a sphere with six punctures, which can be sewn from four pairs of pants in various ways. Two possibilities are shown in Fig. 2 . Both give rise to theories with the gauge group $S U(2)_{1} \times S U(2)_{2} \times S U(2)_{3}$, one for each of the three thin necks with sewing parameter $q_{1,2,3}$. We again denote as $m_{a}$ the 

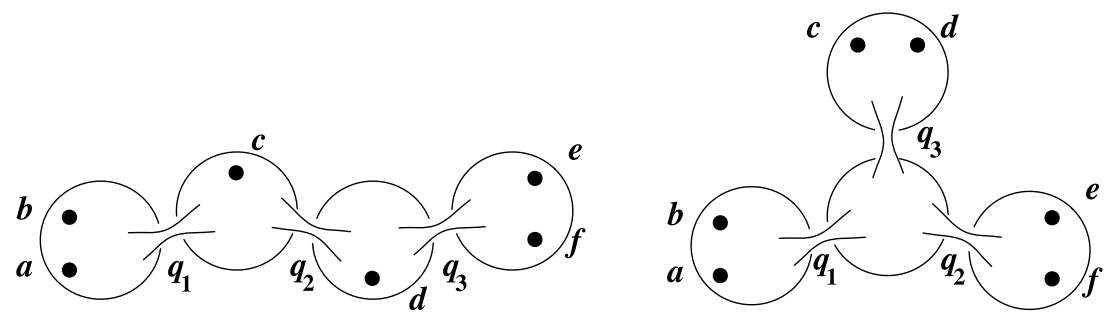

Figure 2: Two examples to sew a six-punctured sphere from four pairs of pants. Left: a standard linear quiver theory. Right: a generalized quiver theory, where three $S U(2)$ gauge groups couple to four hypermultiplets, denoted by the three-punctured sphere at the center.

mass parameter for the $S U(2)_{a}$, etc. Then, the hypermultiplet content of the theory on the left is two fundamentals of $S U(2)_{1}$ with masses $m_{a} \pm m_{b}$, one bifundamental of $S U(2)_{1} \times S U(2)_{2}$ with mass $m_{c}$, another bifundamental of $S U(2)_{2} \times S U(2)_{3}$ with mass $m_{d}$, and two fundamentals of $S U(2)_{1}$ with masses $m_{e} \pm m_{f}$. The matter content of the theory on the right is more exotic: each of $S U(2)_{1,2,3}$ has two fundamentals with masses $m_{a} \pm m_{b}, m_{c} \pm m_{d}$ and $m_{e} \pm m_{f}$, respectively, and the three $S U(2)_{1,2,3}$ all couple a single set of eight $\mathcal{N}=1$ chiral multiplets transforming in $\mathbf{2}_{1} \otimes \mathbf{2}_{2} \otimes \mathbf{2}_{3}$. This is the simplest example of a "generalized quiver" introduced by one of the authors in [1].

In this paper, we will focus on examples where each block of hypermultiplets is either a bifundamental hypermultiplet, or a pair of fundamental hypermultiplets. Hence the "generalized quivers" will simply be linear quivers of $S U(2)$ gauge groups realizing $\mathcal{T}_{0, n}$, or necklace quivers realizing $\mathcal{T}_{1, n}$.

\section{Instanton sums and the conformal blocks}

As we reviewed in the previous section, we can associate an $\mathcal{N}=2$ SCFT to a punctured Riemann surface, and we have a Lagrangian description of this SCFT for each possible way to compose the Riemann surface out of three-punctured spheres.

There are several quantities of interest which can be computed, given the Lagrangian of a four-dimensional field theory. When we combine them with the Lagrangian description for different sewings of a Riemann surface, we have a function on the space of the sewings of the Riemann surface.

We will use below the Nekrasov partition function [2, 3] of the Lagrangian field theory to produce an interesting function; we will see that the resulting function on the space of the sewing is just the standard conformal block of the Virasoro algebra. In order to express our proposal, we need to review briefly what the Nekrasov partition function is. 


\subsection{Nekrasov's partition function}

For a given four-dimensional $\mathcal{N}=2$ field theory, Nekrasov considered a deformation of its Lagrangian by two deformation parameters $\epsilon_{1,2}$ parameterizing the $S O(4)$ rotation of the spacetime $\mathbb{R}^{4}$. This breaks the translational symmetry of the system. The partition function is just a number, which depends meromorphically on the coupling constants $\tau$, vevs $a$ of the adjoint scalars in the vector multiplets, and hypermultiplet masses $m$. The Nekrasov partition function consists of three parts: the classical, the one-loop, and the instanton parts:

$$
Z_{\text {full }}\left(\tau, a, m ; \epsilon_{i}\right)=Z_{\text {classical }} Z_{1-\text { loop }} Z_{\text {instanton }}
$$

It has the important property that it gives the prepotential of the theory in the limit $\epsilon_{1,2} \rightarrow 0$

$$
F(\tau, a, m)=\lim _{\hbar \rightarrow 0} \hbar^{2} \log Z_{\text {full }}(\tau, a, m ; \hbar,-\hbar) .
$$

This limit was evaluated for a number of $\mathcal{N}=2$ theories, and reproduced the prepotential as determined by the Seiberg-Witten curve.

The path integral of the Lagrangian deformed by $\epsilon_{1,2}$ localizes to instanton configurations sitting at the origin of the spacetime $\mathbb{R}^{4}$. For $U(N)$ gauge group, such instantons are labeled by an $N$-tuple of Young tableaux $\vec{Y}=\left(Y_{1}, \ldots, Y_{N}\right)$. The instanton number of the configuration is given by the number of the boxes $|\vec{Y}|$.

Then the instanton part of the Nekrasov partition function is the summation over the Young tableaux, whose summand is the product of factors corresponding to the field content of the Lagrangian. As an example, we give the expression for $U(2)$ gauge theory with one fundamental hypermultiplet:

$$
Z_{\mathrm{inst}}^{U(2), N_{f}=1}(q, \vec{a}, m)=\sum_{\vec{Y}} q^{|\vec{Y}|} z_{\text {vector }}(\vec{a}, \vec{Y}) z_{\text {fund }}(\vec{a}, \vec{Y}, m),
$$

where $\vec{a}=\left(a_{1}, a_{2}\right)$ is the vev of the adjoint scalar and $m$ is the mass of the hypermultiplet. The explicit form of $z_{\text {vector }}$ and $z_{\text {fund }}$ can be found in Appendix $\mathbb{B}$.

There are two subtleties of Nekrasov's formulation which will complicate our investigation. One is that the deformation by $\epsilon_{i}$ treats hypermultiplets in complex conjugate representations $R$ and $R^{*}$ differently, so that the contribution of a hypermultiplet in the representation $R$ with mass $m$ is equivalent to that of another hypermultiplet in $R^{*}$ with mass $\epsilon_{1}+\epsilon_{2}-m$ :

$$
z_{R}(m)=z_{R^{*}}\left(\epsilon_{1}+\epsilon_{2}-m\right)
$$

The other is that we will use Nekrasov's partition function for $U(2)$ quiver theories, not for $S U(2)$ quiver theories. For $U(2)$, the doublet and the anti-doublet are two distinct representations. Therefore, the expression (3.3) above does not have the

symmetry under $m \rightarrow \epsilon_{1}+\epsilon_{2}-m$ which should be there for $S U(2)$ gauge theory. 
We propose to remedy this situation by decoupling the contribution of $U(1)$ gauge fields from the instanton partition function so that the symmetry under $m \rightarrow$ $\epsilon_{1}+\epsilon_{2}-m$ is recovered. We find it rather nontrivial that the decoupling can be consistently performed at all.

\subsection{Sphere with four punctures}

Here and in the following, we will deal with superconformal theories. The deformation parameters $\epsilon_{i}$, the vevs $a$ and the masses $m_{i}$ all have mass dimension one. We choose to fix the scale by setting

$$
\epsilon_{1}=b, \quad \epsilon_{2}=1 / b
$$

We also define

$$
Q=\epsilon_{1}+\epsilon_{2}=b+1 / b .
$$

Let us consider the simplest case, the six-dimensional $(2,0)$ theory of type $A_{1}$ compactified on a sphere with four punctures. The manifest flavor symmetry in this description is $S U(2)^{4}$, one $S U(2)$ factor for each puncture. At low energy, this becomes $\mathcal{N}=2 S U(2)$ gauge theory with $N_{f}=4$ flavors and the flavor symmetry enhances to $S O(8)$.

We write down Nekrasov's instanton partition function for $U(2)$ theory with $N_{f}=4$ flavors instead, which is given by the formula

$$
\begin{aligned}
Z_{\text {inst }}^{U(2), N_{f}=4}= & \sum_{\vec{Y}} q^{|\vec{Y}|} z_{\text {vector }}(\vec{a}, \vec{Y}) \\
& z_{\text {antifund }}\left(\vec{a}, \vec{Y}, \mu_{1}\right) z_{\text {antifund }}\left(\vec{a}, \vec{Y}, \mu_{2}\right) z_{\text {fund }}\left(\vec{a}, \vec{Y}, \mu_{3}\right) z_{\text {fund }}\left(\vec{a}, \vec{Y}, \mu_{4}\right) .
\end{aligned}
$$

Here $\vec{a}=\left(a_{1}, a_{2}\right)$ is the adjoint vev of the $U(2)$ gauge multiplet, $\mu_{1,2}$ are the masses of two hypermultiplets in the anti-fundamental, and $\mu_{3,4}$ are those of the fundamentals. Explicit expressions for the contributions $z_{\text {vector,fund,antifund }}$ can be found in Appendix B.

Manifest flavor symmetries are now $U(2)_{1} \times U(2)_{2}$, acting on $\mu_{1,2}$ and $\mu_{3,4}$, respectively. We redefine them as follows:

$$
\mu_{1}=m_{0}+\tilde{m}_{0}, \quad \mu_{2}=m_{0}-\tilde{m}_{0}, \quad \mu_{3}=m_{1}+\tilde{m}_{1}, \quad \mu_{4}=m_{1}-\tilde{m}_{1} .
$$

$m_{i}$ is the mass parameter associated to $U(1)_{i} \subset U(2)_{i}$, and $\tilde{m}_{i}$ is the one associated to $S U(2)_{i} \subset U(2)_{i}$.

Let us stress that the formula (3.7) is for $U(2)$ gauge group; one expects that by decoupling the $U(1)$ part of the gauge group the manifest flavor symmetry $U(2)_{i}=$ $S U(2)_{i} \times U(1)_{i}$ would enhance to $S U(2)_{i} \times S U(2)_{i}$. To do that, one needs to set $\vec{a}=\left(a_{1}, a_{2}\right)=(a,-a)$, and also to eliminate the contribution from the $U(1)$ gauge multiplet. Without further ado, we propose how to decouple the $U(1)$ part:

$$
Z_{\text {inst }}^{U(2), N_{f}=4}\left(a, m_{0}, \tilde{m}_{0}, m_{1}, \tilde{m}_{1}\right)=(1-q)^{2 m_{0}\left(Q-m_{1}\right)} \mathcal{F}_{\alpha_{0}}^{m_{0}{ }_{\alpha}{ }^{m_{1}}{ }_{\alpha_{1}}(q)}
$$




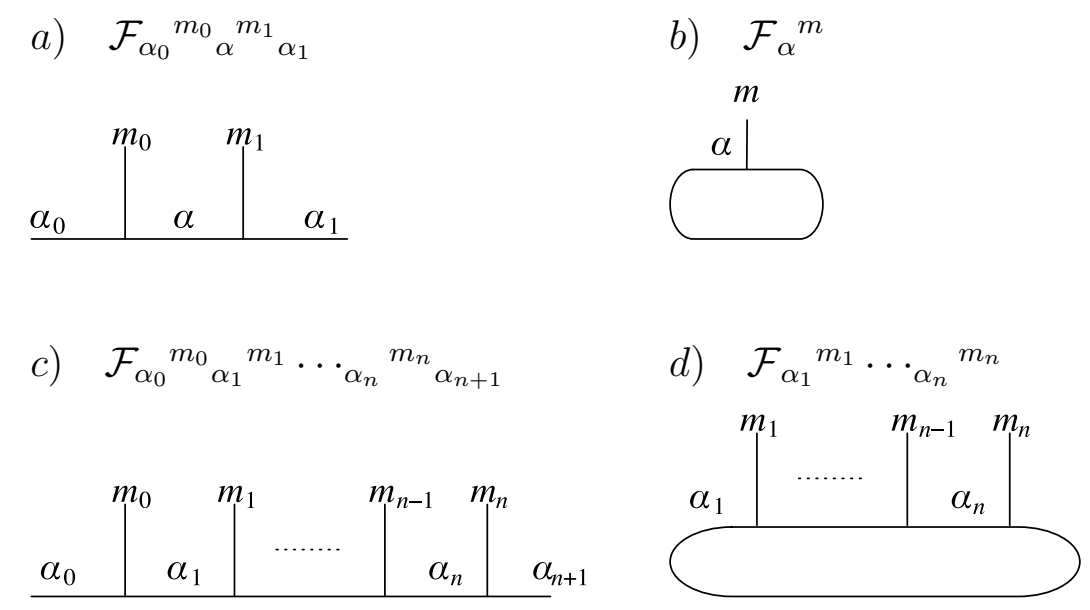

Figure 3: Placement of labels of the conformal blocks we use.

where the relation between $\alpha, \alpha_{i}$ and the mass parameters is given by

$$
\alpha=Q / 2+a, \quad \alpha_{0}=Q / 2+\tilde{m}_{0}, \quad \alpha_{1}=Q / 2+\tilde{m}_{1} .
$$

By explicit calculation, one can check that $\mathcal{F}_{\alpha_{0}}{ }^{m_{0}}{ }_{\alpha}{ }^{m_{1}}{ }_{\alpha_{1}}(q)$ is a function invariant under individual flips of $\alpha, \alpha_{i}$, and $m_{i}$ :

$$
\alpha \rightarrow Q-\alpha, \quad \alpha_{i} \rightarrow Q-\alpha_{i}, \quad m_{i} \rightarrow Q-m_{i}
$$

We identify the flip of $\alpha$ as the action of the Weyl group of the $S U(2)$ gauge symmetry, and the flips of $\alpha_{i}, m_{i}$ as that of the four $S U(2)$ flavor symmetries. The prefactor $(1-q)^{2 m_{0}\left(Q-m_{1}\right)}$ is not invariant under the flip of $m_{0,1}$, but is independent of $a$, which is the expected property for the contribution of the $U(1)$ gauge field.

Now we come to a surprising observation: explicit calculation ${ }^{1}$ tells us that $\mathcal{F}_{\alpha_{0}}{ }^{m_{0}}{ }_{\alpha}{ }^{m_{1}}{ }_{\alpha_{1}}(q)$ is exactly the conformal block of the Virasoro algebra with central charge $c=1+6 Q^{2}$ for four operators of dimensions $\Delta_{1,2,3,4}$ inserted at $\infty, 1, q, 0$, respectively and with an intermediate state in the $s$-channel whose dimension is $\Delta$, see Figure 3 a). Here

$$
\begin{aligned}
& \Delta=\alpha(Q-\alpha), \quad \Delta_{1}=\alpha_{0}\left(Q-\alpha_{0}\right), \quad \Delta_{2}=m_{0}\left(Q-m_{0}\right), \\
& \Delta_{3}=m_{1}\left(Q-m_{1}\right), \quad \Delta_{4}=\alpha_{1}\left(Q-\alpha_{1}\right) \text {. }
\end{aligned}
$$

Note that $\mathcal{F}_{\alpha_{0}}{ }^{m_{0}}{ }_{\alpha}{ }^{m_{1}}{ }_{\alpha_{1}}(q)$ does not have the symmetry under the permutation of $\alpha_{0,1}$ and $m_{0,1}$ keeping $q$ fixed. In other words, it is not invariant under $S O(8)$ keeping $q$ fixed. It should not come as a surprise, because even the Seiberg-Witten curve of this theory in the formalism reviewed in Sec. 2 does not have the manifest $S O(8)$ symmetry.

\footnotetext{
${ }^{1}$ We checked this statement up to order $q^{11}$ in the instanton expansion.
} 


\subsection{Torus with one puncture}

The second simplest example is the $\mathcal{N}=2^{*}$ theory, i.e. $\mathcal{N}=4 S U(2)$ theory deformed by a mass term for the adjoint hypermultiplet. For the $\mathcal{N}=2^{*} U(2)$ theory, Nekrasov's instanton partition function is

$$
Z_{\text {inst }}^{\mathcal{N}=2^{*}, U(2)}=\sum_{\vec{Y}} q^{|\vec{Y}|} z_{\text {vector }}(\vec{a}, \vec{Y}) z_{\text {adj }}(\vec{a}, \vec{Y}, m) .
$$

This is invariant under the flip $m \rightarrow Q-m$. Encouraged by our observation in the previous subsection, we might hope that we would get a conformal block by setting $\vec{a}=\left(a_{1}, a_{2}\right)=(a,-a)$ and splitting off the contribution from the $U(1)$ vector multiplet. Indeed, by some trial and error one finds

$$
Z_{\mathrm{inst}}^{\mathcal{N}=2^{*}, U(2)}(q, a, m)=\left[\prod_{i=1}^{\infty}\left(1-q^{i}\right)\right]^{-1+2 m(Q-m)} \mathcal{F}_{\alpha}{ }^{m}(q),
$$

where $\mathcal{F}_{\alpha}{ }^{m}(q)$ is the conformal block of the Virasoro algebra of central charge $c=$ $1+6 Q^{2}$ on a torus whose modulus is $q$, with one operator of dimension $\Delta_{1}=m(Q-m)$ inserted and a primary of dimension $\Delta=\alpha(Q-\alpha)$ in the intermediate channel ${ }^{2}$; here $\alpha=Q / 2+a$ as before. See Figure $3 \mathrm{~b}$ ).

\subsection{Sphere with multiple punctures}

Now let us move on to more complicated cases. A sphere with $n+3$ punctures has a weakly-coupled description as a linear quiver gauge theory with $n S U(2)_{i}$ gauge groups, $i=1, \ldots, n$, with coupling constants $q_{i}$ and the vevs $a_{i}$. The hypermultiplets are two antifundamentals of mass $\mu_{1,2}$ on $S U(2)_{1}$, one bifundamental of mass $m_{i}$ between $S U(2)_{i}$ and $S U(2)_{i+1}$, and two fundamentals of mass $\mu_{3,4}$ on $S U(2)_{n}$.

It is straightforward to write down Nekrasov's instanton sum for the quivers of $U(2)$ gauge groups. Again it is natural to rewrite the masses of (anti)-fundamentals as

$$
\mu_{1}=m_{0}+\tilde{m}_{0}, \quad \mu_{2}=m_{0}-\tilde{m}_{0}, \quad \mu_{3}=m_{n}+\tilde{m}_{1}, \quad \mu_{4}=m_{n}-\tilde{m}_{1} .
$$

We propose to decouple the $U(1)$ factor in the following way:

$$
\begin{aligned}
& Z_{\text {inst }}^{U(2) \text { linear quiver }}\left(q_{i} ; a_{i} ; m_{i} ; \tilde{m}_{i}\right) \\
& \quad=Z^{U(1) \text { linear }}\left(q_{i} ; m_{i}\right) \mathcal{F}_{\alpha_{0}}{ }^{m_{0}}{ }_{\alpha_{1}}{ }^{m_{1}} \cdots{ }_{\alpha_{n}}{ }^{m_{n}}{ }_{\alpha_{n+1}}\left(q_{1}, q_{2}, \ldots, q_{n}\right)
\end{aligned}
$$

where

$$
\alpha_{0}=Q / 2+\tilde{m}_{0}, \quad \alpha_{i}=Q / 2+a_{i}, \quad \alpha_{n+1}=Q / 2+\tilde{m}_{1} .
$$

\footnotetext{
${ }^{2}$ We have checked this up to order $q^{7}$ in the instanton expansion.
} 
The $U(1)$ factor $Z^{U(1)}$ linear $\left(q_{i} ; m_{i}\right)$ is detailed in App. C; this is a function only of the coupling constants $q_{i}$ and the masses $m_{i}$, and is not symmetric under $m_{i} \rightarrow Q-m_{i}$.

$\mathcal{F}_{\alpha_{0}}{ }^{m_{0}}{ }_{\alpha_{1}} m_{1} \ldots{ }_{\alpha_{n}}{ }^{m_{n}}{ }_{\alpha_{n+1}}\left(q_{1}, q_{2}, \ldots, q_{n}\right)$ can then be seen to be the conformal block of Virasoro algebra with central charge $c=1+6 Q^{2}$ for a sphere with $n+3$ punctures at

$$
\infty, 1, q_{1}, q_{1} q_{2}, \ldots, q_{1} q_{2} \cdots q_{n}, 0
$$

The dimensions of the operators at the punctures are

$$
\alpha_{0}\left(Q-\alpha_{0}\right), m_{0}\left(Q-m_{0}\right), \ldots, m_{n}\left(Q-m_{n}\right), \alpha_{n+1}\left(Q-\alpha_{n+1}\right)
$$

respectively, and that of the operator in the $i$-th intermediate channel is $\alpha_{i}\left(Q-\alpha_{i}\right)$, see Figure 3 c).

\subsection{Torus with multiple punctures}

Let us next consider a torus with $n$ points. It has a weakly-coupled description as a necklace quiver gauge theory with $n S U(2)_{i}$ gauge groups, $i=1, \ldots, n$, with coupling constants $q_{i}$ and the vevs $a_{i}$. The hypermultiplets are one bifundamental of mass $m_{i}$ between each consecutive pair of gauge groups $S U(2)_{i}$ and $S U(2)_{i+1}$; we identify $S U(2)_{n+1}$ with $S U(2)_{1}$.

It is again straightforward to write down Nekrasov's instanton sum for the quivers of $U(2)$ gauge groups. We propose to decouple the $U(1)$ factor in the following way:

$$
\begin{aligned}
& Z_{\text {inst }}^{U(2) \text { necklace quiver }}\left(q_{i} ; a_{i} ; m_{i}\right) \\
& \quad=Z^{U(1) \text { necklace }}\left(q_{i} ; m_{i}\right) \mathcal{F}_{\alpha_{1}}^{m_{1}} \cdots_{\alpha_{n}}^{m_{n}}\left(q_{1}, q_{2}, \ldots, q_{n}\right)
\end{aligned}
$$

where $\alpha_{i}=Q / 2+a_{i}$. The $U(1)$ factor is again detailed in App. Q; this is a function only of the coupling constants $q_{i}$ and the masses $m_{i}$, and is not symmetric under $m_{i} \rightarrow Q-m_{i}$ in general.

$\mathcal{F}_{\alpha_{1}} m_{1} \ldots \alpha_{n} m_{n}\left(q_{1}, q_{2}, \ldots, q_{n}\right)$ can then be seen to be the conformal block of Vi-

rasoro algebra with central charge $c=1+6 Q^{2}$ for a torus with modulus $q_{1} q_{2} \cdots q_{n}$ with punctures at

$$
1, q_{1}, q_{1} q_{2}, \ldots, q_{1} q_{2} \cdots q_{n-1} .
$$

The dimension of the operator at the $i$-th puncture is $m_{i}\left(Q-m_{i}\right)$, and that of the operator in the $i$-th intermediate channel is $\alpha_{i}\left(Q-\alpha_{i}\right)$, see Figure $\left.3 \mathrm{~d}\right)$.

\section{Liouville correlators}

In the last section we presented a concrete way to decouple the $U(1)$ part from Nekrasov's instanton partition function for $U(2)$ quivers. This resulted in the identification of the $S U(2)$ part of the instanton sum with the Virasoro conformal block with central charge $c=1+6 Q^{2}$. 
Conformal blocks, combined with three-point functions, give multi-point correlators of concrete CFTs. This then begs a natural question: is there something we need to combine with the instanton part of Nekrasov's partition function, to form another physical quantity? Indeed there is such a thing, which is the one-loop part of Nekrasov's partition function.

From the point of view of the low-energy theory, splitting the prepotential into the one-loop and the instanton parts is rather artificial in that the split depends on the electromagnetic frame one is interested in. This is analogous to the situation with the multi-point correlators of a $2 \mathrm{~d}$ CFT: decomposition of the correlators into the products of three-point functions and conformal blocks depends on the channel one is interested in.

We will show below that the one-loop factor precisely reproduces the product of the DOZZ three-point functions [5, 6, 7] of the Liouville theory. In other words, the absolute value squared of the full Nekrasov partition function, integrated over the vevs $a_{i}$, is a Liouville correlator.

\subsection{Sphere with four punctures}

Without further ado, let us consider the four-point function of the Liouville theory on a sphere,

$$
\begin{aligned}
& \left\langle V_{\alpha_{0}}(\infty) V_{m_{0}}(1) V_{m_{1}}(q) V_{\alpha_{1}}(0)\right\rangle \\
& \quad=\int \frac{d \alpha}{2 \pi} C\left(\alpha_{0}^{*}, m_{0}, \alpha\right) C\left(\alpha^{*}, m_{1}, \alpha_{1}\right) \mid q^{\Delta_{\alpha}-\Delta_{m_{1}}-\Delta_{\alpha_{1}}} \mathcal{F}_{\alpha_{0}}{ }^{\left.m_{0}{ }_{\alpha}{ }^{m_{1}}{ }_{\alpha_{1}}(q)\right|^{2}}
\end{aligned}
$$

Here and in the following, $V_{\alpha}(z)=e^{2 \alpha \phi(z)}$ is the Liouville exponential, and we take all $\alpha, \alpha_{i}, m_{i}$ to be $\in Q / 2+i \mathbb{R}$; the integral over $\alpha$ is along this line. We also use the notation $\alpha=Q / 2+a, \alpha_{i}=Q / 2+\tilde{m}_{i}, m_{i}=Q / 2+\hat{m}_{i}$. The threepoint function $C\left(\alpha_{1}, \alpha_{2}, \alpha_{3}\right)$ is given by the DOZZ formula, see Appendix A. Using formulae collected there, one can massage the right hand side into the form

$$
=f\left(\alpha_{0}^{*}\right) f\left(m_{0}\right) f\left(m_{1}\right) f\left(\alpha_{1}\right)\left|q^{Q^{2} / 4-\Delta_{m_{1}}-\Delta_{\alpha_{1}}}\right|^{2} \int a^{2} d a \mid Z_{\alpha_{0}}^{\left.m_{0}{ }_{\alpha}{ }^{m_{1}}{ }_{\alpha_{1}}(q)\right|^{2}}
$$

up to a constant which only depends on $b$. Here

$$
f(\alpha)=\left[\pi \mu \gamma\left(b^{2}\right) b^{2-2 b^{2}}\right]^{-\alpha / b} \Upsilon(2 \alpha)
$$

and

$$
\begin{aligned}
& Z_{\alpha_{0}}{ }^{m_{0}}{ }_{\alpha}{ }^{m_{1}}{ }_{\alpha_{1}}(q)= \\
& q^{-a^{2}} \frac{\prod \Gamma_{2}\left(\hat{m}_{0} \pm \tilde{m}_{0} \pm a+Q / 2\right) \prod \Gamma_{2}\left(\hat{m}_{1} \pm \tilde{m}_{1} \pm a+Q / 2\right)}{\Gamma_{2}(2 a+b) \Gamma_{2}(2 a+1 / b)} \mathcal{F}_{\alpha_{0}}{ }^{m_{0}}{ }_{\alpha}{ }^{m_{1}}{ }_{\alpha_{1}}(q) .
\end{aligned}
$$


In the last expression each product is over the four choices of signs. Using the formula for the one-loop factors collected in Appendix B, ${ }^{3}$ we find it is equal to

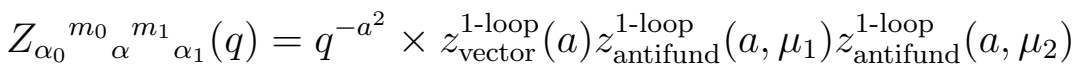

$$
\begin{aligned}
& \times z_{\text {fund }}^{1 \text {-loop }}\left(a, \mu_{3}\right) z_{\text {fund }}^{1 \text {-loop }}\left(a, \mu_{4}\right) \mathcal{F}_{\alpha_{0}}{ }^{m_{0}{ }_{\alpha}{ }^{m_{1}}{ }_{\alpha_{1}}(q)}
\end{aligned}
$$

where

$$
\mu_{1}=m_{0}+\tilde{m}_{0}, \quad \mu_{2}=m_{0}-\tilde{m}_{0}, \quad \mu_{3}=m_{1}+\tilde{m}_{1}, \quad \mu_{4}=m_{1}-\tilde{m}_{1} .
$$

We identified in the previous section $\mathcal{F}_{\alpha_{0}}{ }^{m_{0}}{ }_{\alpha}{ }^{m_{1}}{ }_{\alpha_{1}}(q)$ as Nekrasov's instanton partition function for $S U(2)$ theory with four flavors with masses $\mu_{1,2}$ and $\mu_{3,4}$. One can easily see that $q^{-a^{2}}$ gives the exponential of the classical prepotential $(2 \pi i) \tau_{\mathrm{UV}} a^{2}$, and the product of the one-loop factors is exactly the one for this gauge theory. Thus, $Z_{\alpha_{0}}{ }^{m_{0}}{ }_{\alpha}{ }^{m_{1}}{ }_{\alpha_{1}}(q)$ is precisely Nekrasov's full partition function of $S U(2)$ gauge theory with four flavors. In the integral (4.2), the absolute value squared of this partition function is integrated over the natural measure $a^{2} d a$ on the Cartan subalgebra of $S U(2)$, including the Vandermonde determinant. Therefore, we have come to a surprising conclusion that Nekrasov's full partition function, integrated over the vev with the natural measure, is the Liouville four-point function on the sphere.

This integral, from the gauge theory point of view, has appeared in [4] when $b=1 / b=1$. There, the integral (4.2) without the prefactor, i.e.

$$
\int a^{2} d a \mid Z_{\alpha_{0}}^{\left.m_{0}{ }_{\alpha}^{m_{1}}{ }_{\alpha_{1}}(q)\right|^{2}}
$$

appeared as the partition function of the $4 \mathrm{~d}$ SCFT on $S^{4}$.

The Liouville four-point function, as constructed from the DOZZ three-point functions and the conformal blocks, has been proved in [13, 14] to be crossing symmetric. Therefore, we find that the absolute-value squared of Nekrasov's partition function, integrated over the vev $a$, is indeed an S-duality invariant object.

\subsection{Torus with one puncture}

We can perform the same procedure on the one-point function of the Liouville theory on a torus:

$$
\begin{aligned}
\left\langle V_{m}\right\rangle_{q} & =\int \frac{d \alpha}{2 \pi} C\left(\alpha^{*}, m, \alpha\right)\left|q^{\Delta_{\alpha}} \mathcal{F}_{\alpha}{ }^{m}(q)\right|^{2} \\
& =c^{\prime} f(m) \int a^{2} d a\left|Z_{\alpha}{ }^{m}(q)\right|^{2}
\end{aligned}
$$

\footnotetext{
${ }^{3}$ As will be elaborated there, our one-loop factor for the vector multiplet is slightly different from that in [3], but agrees with that in [11, 12].
} 
where

$$
Z_{\alpha}{ }^{m}(q)=q^{-a^{2}} z_{\text {vector }}^{1 \text {-loop }}(a) z_{\text {adj }}^{1 \text {-loop }}(a, m) \mathcal{F}_{\alpha}{ }^{m}(q) .
$$

We have identified in the previous section $\mathcal{F}_{\alpha}{ }^{m}(q)$ as the instanton part of Nekrasov's partition function of $\mathcal{N}=2^{*} S U(2)$ gauge theory. The DOZZ formula gave us precisely the one-loop factors for the vector multiplet and the adjoint hypermultiplet, and thus $Z_{\alpha}{ }^{m}(q)$ is Nekrasov's full partition function of this gauge theory. Therefore, we find that the one-point function of the Liouville theory on a torus is the absolute value squared of Nekrasov's full partition function, integrated over $a$ with the natural measure.

The modular invariance of the torus one-point function of the Liouville theory, as constructed from the DOZZ three-point function and the conformal block, has not been fully demonstrated yet, but presumably it can be shown using the equivalence of the Liouville theory to the quantum Teichmüller theory [15, 16, 17]. It would be worthwhile to prove the modular invariance of $(4.9)^{4}$, which then implies the S-duality of the $\mathcal{N}=2^{*}$ theory.

The one-loop factor cancels when $m=0$ and Eq.(4.9) reproduces the standard torus amplitude of the Liouville theory. This limit corresponds to the $\mathcal{N}=4 S U(2)$ theory. ${ }^{5}$

\subsection{General proposal}

The generalization of the analysis above to multiple points on a sphere and on a torus is now immediate. A sphere with $n+3$ punctures corresponds to a linear quiver of $n S U(2)$ gauge groups. Then we have the relation

$$
\begin{aligned}
& \left\langle V_{\alpha_{0}}(\infty) V_{m_{0}}(1) V_{m_{1}}\left(q_{1}\right) \cdots V_{m_{n}}\left(q_{1} \cdots q_{n}\right) V_{\alpha_{n+1}}(0)\right\rangle= \\
& c f\left(\alpha_{0}\right) f\left(\alpha_{n+1}\right) \prod f\left(m_{i}\right) \int \prod\left(a_{i}^{2} d a_{i}\right)\left|Z_{\alpha_{0}}{ }^{m_{0}}{ }_{\alpha_{1}}{ }^{m_{1}} \cdots_{\alpha_{n}}{ }^{m_{n}}{ }_{\alpha_{n+1}}\left(q_{i}\right)\right|^{2}
\end{aligned}
$$

where $Z_{\alpha_{0}}{ }^{m_{0}}{ }_{\alpha_{1}} m_{1} \cdots{ }_{\alpha_{n}}{ }^{m_{n}}{ }_{\alpha_{n+1}}\left(q_{i}\right)$ is the Nekrasov's full partition function for this $S U(2)$ quiver gauge theory, i.e. $\mathcal{F}_{\alpha_{0}}{ }^{m_{0}}{ }_{\alpha_{1}} m_{1} \cdots_{\alpha_{n}}{ }^{m_{n}}{ }_{\alpha_{n+1}}\left(q_{i}\right)$ multiplied by the one-loop factors from the vector and hypermultiplets. The relation for the $n$-point function on a torus can be written down in a similar manner.

The rewriting of the product of the DOZZ three-point functions as the one-loop factor is analogous to what was presented above for $S U(2) N_{f}=4$ theory and $\mathcal{N}=2^{*}$ $S U(2)$ theory, so we just mention two salient points. Consider a three-point function

\footnotetext{
${ }^{4}$ This was achieved in [18] in November 2009.

${ }^{5}$ The partition function was calculated in 顿. There, the one-loop factor of the vector multiplet used was slightly different from ours, as we will explain in more detail in Appendix B.2. This difference produces extra powers of $\operatorname{Im} \tau$, effectively replacing his $|\eta(\tau)|^{2}$ by $\sqrt{\operatorname{Im} \tau}|\eta(\tau)|^{2}$. This makes the partition function modular invariant. See also [19].
} 
$C\left(Q-\alpha_{1}, m, \alpha_{2}\right)$. The denominator of the DOZZ formula then gives

$$
\prod \Gamma_{2}\left( \pm a_{1} \pm a_{2} \pm \hat{m}+\frac{Q}{2}\right)
$$

where $\alpha_{i}=Q / 2+a_{i}, m=Q / 2+\hat{m}$. This is the absolute value squared of the one-loop contribution of a bifundamental hypermultiplet of mass $m$, charged under two $S U(2)$ gauge groups, with vevs $\pm a_{1}$ and $\pm a_{2}$ respectively. Next consider the numerator of the DOZZ formula. When we glue two three-point functions along the channel where $V_{\alpha}$ is inserted, we have the product of the form $C(\bullet, \bullet, \alpha) C(Q-\alpha, \bullet, \bullet)$. Then the numerator of the DOZZ formula gives

$$
\begin{aligned}
\Upsilon(2(Q-\alpha)) \Upsilon(2 \alpha) & =\left[\Gamma_{2}(2 a) \Gamma_{2}(2 a+Q) \Gamma_{2}(-2 a) \Gamma_{2}(-2 a+Q)\right]^{-1} \\
& =-4 a^{2} \prod_{i=1,2}\left[\Gamma_{2}\left(2 a+\epsilon_{i}\right) \Gamma_{2}\left(-2 a+\epsilon_{i}\right)\right]^{-1}
\end{aligned}
$$

where we used the formula (A.14). This gives the absolute value squared of the contribution from the $S U(2)$ vector multiplet with the vev $a$, and also provides the crucial Vandermonde factor $a^{2}$.

\section{Seiberg-Witten differential and the insertion of $T(z)$}

Conformal blocks are only a fragment of a full CFT correlation function, but, almost by construction, satisfy an important property: Ward identities for the insertion of energy momentum tensor operators. This insertion can be defined directly by inserting the power expansion of the operator

$$
T(z)=\sum L_{n} z^{-n-2}
$$

anywhere in the definition of the conformal block, or can be simply computed through the Ward identity. On the sphere, for example,

$$
\left\langle T(z) \prod_{i} \mathcal{O}_{i}\left(z_{i}\right)\right\rangle=\sum_{j}\left[\frac{h_{j}}{\left(z-z_{j}\right)^{2}}+\frac{\partial_{j}}{z-z_{j}}\right]\left\langle\prod_{i} \mathcal{O}_{i}\left(z_{i}\right)\right\rangle
$$

where the insertion is made at the level of the conformal block. Do such energy momentum tensor insertions have any interesting meaning in the gauge theory side? We can define a useful quadratic differential

$$
\phi_{2}(z) d z^{2}=-\frac{\left\langle T(z) \prod_{i} \mathcal{O}_{i}\left(z_{i}\right)\right\rangle}{\left\langle\prod_{i} \mathcal{O}_{i}\left(z_{i}\right)\right\rangle}
$$

$\phi_{2}(z)$ has double poles at $z_{i}$ with coefficient $-h_{i}$. The space of quadratic differentials with double poles of fixed coefficients is an affine space of dimension $3 g-3+n$. This is also the dimension of the Coulomb branch of $\mathcal{T}_{g, n}$. Indeed the Seiberg-Witten curve 
of the theory can be also written as a double cover of $C_{g, n}$, in terms of a quadratic differential $\phi_{2}^{S W}(z)$, as

$$
x^{2}=\phi_{2}^{S W}(z)
$$

The coefficients of the double poles of $\phi_{2}^{S W}(z)$ are the squared mass parameters $-m_{i}^{2}$, i.e.

$$
m_{a}=\frac{1}{2 \pi i} \oint_{\beta_{a}} x d z
$$

where $\beta_{a}$ is a small circle around the $a$-th puncture. The other $3 g-3+n$ moduli can be fixed in terms of the special coordinates $a_{i}$ by computing the electric periods of the Seiberg-Witten differential

$$
a_{i}=\frac{1}{2 \pi i} \oint_{\gamma_{i}} x d z
$$

The cycles $\gamma_{i}$ are defined at weak coupling as wrapping around the long $i$-th tube.

The Seiberg-Witten curve is supposed to emerge from the Nekrasov partition function in the "semiclassical limit" $\epsilon_{1,2} \ll a_{i}, m_{i}$. We expect that

$$
\phi_{2}(z) \rightarrow \phi_{2}^{S W}(z)
$$

in the same limit. The property (5.5) can be easily checked: we have

$$
\frac{1}{2 \pi i} \oint_{\beta_{a}} \sqrt{\phi_{2}(z)}=\sqrt{-h_{a}} \rightarrow m_{a} .
$$

Here we used (5.3) in the first equality, and $h_{a}=m_{a}\left(Q-m_{a}\right)$ where $Q=\epsilon_{1}+\epsilon_{2}$ in the second limit. We have also checked that

$$
\frac{1}{2 \pi i} \oint_{\gamma_{i}} \sqrt{\phi_{2}(z)} \rightarrow a_{i}
$$

to high order in the expansion of the conformal blocks for $\mathcal{T}_{0,4}, \mathcal{T}_{0,5}, \mathcal{T}_{1,0}$ and $\mathcal{T}_{1,0}$. The agreement is quite remarkable, as the coefficients of $\phi_{2}$ and $\phi_{2}^{S W}$ are very intricate functions of $a_{i}, m_{i}, \tau_{i}$. We expect this to be true for all $g, n$.

We are then led to speculate that at finite $\epsilon_{1,2}$, the notion of Seiberg-Witten curve should be "quantized" to the operator equation $x^{2}+T(z)=0$.

\section{Conclusions and Open Problems}

In this paper we considered Nekrasov's partition function of four-dimensional $\mathcal{N}=2$ theories which arise from compactification of six-dimensional $(2,0)$ theory of type $A_{1}$ on a sphere or a torus with punctures. We showed that the instanton part of the partition function gives the conformal blocks, and the one-loop part gives the

products of the DOZZ three-point functions of the Liouville theory. Therefore, the 
integral of Nekrasov's full partition function over the vevs of the adjoint scalars gives the Liouville correlation functions.

With these observations at hand, we propose the following general statement: Given a genus- $g$ Riemann surface with $n$ punctures and a particular sewing of the surface from three-punctured spheres, consider the generalized quiver gauge theory naturally associated to it. Then, the conformal block for this sewing is the instanton part of Nekrasov's partition function of this gauge theory. Furthermore, the n-point function of the Liouville theory on this Riemann surface is equal to the integral of the absolute value squared of Nekrasov's full partition function of this gauge theory. The dictionary between the two sides of the correspondence is summarized in Table 1 .

\begin{tabular}{||c|c||}
\hline Gauge theory & Liouville theory \\
\hline \hline Deformation parameters $\epsilon_{1}, \epsilon_{2}$ & Liouville parameters \\
& $\epsilon_{1}: \epsilon_{2}=b: 1 / b$ \\
\hline four free hypermultiplets & a three-punctured sphere \\
\hline Mass parameter $m$ & Insertion of \\
associated to an $S U(2)$ flavor & a Liouville exponential $e^{2 m \phi}$ \\
\hline one $S U(2)$ gauge group & a thin neck (or channel) \\
with UV coupling $\tau$ & with sewing parameter $q=$ exp $(2 \pi i \tau)$ \\
\hline Vacuum expectation value $a$ & Primary $e^{2 \alpha \phi}$ for the channel, \\
of an $S U(2)$ gauge group & $\alpha=Q / 2+a$ \\
\hline Instanton part of $Z$ & Conformal blocks \\
\hline One-loop part of $Z$ & Product of DOZZ factors \\
\hline Integral of $\left|Z_{\text {full }}^{2}\right|$ & Liouville correlator \\
\hline \hline
\end{tabular}

Table 1: Dictionary between the Liouville correlation functions and Nekrasov's partition function $Z$.

There are many open problems which beg to be answered. We list them in a random order:

1. Prove mathematically the relation between conformal blocks and Nekrasov's partition function of the $U(2)$ quiver theory, stripped of the $U(1)$ part. Techniques developed in [20, 21] might be useful.

2. Understand better the $U(1)$ part which we stripped manually. 
3. Our identification of Nekrasov's instanton partition function and the conformal block tells us how the former transforms under the S-duality, in terms of the crossing symmetry of the conformal block. Find a physical explanation of this transformation law. cf. [22].

4. What is the relation of the Liouville theory and the theory of chiral bosons ubiquitous in the topological vertex? cf. [23, 24].

5. We found that Liouville correlators are the integral of the absolute value squared of Nekrasov's full partition function. This should be related to the OSV conjecture [25]. Make the relation precise.

6. Calculate directly Nekrasov's partition function for $S U(2)$ quivers, rather than for $U(2)$ quivers. This should be possible by treating $S U(2)$ as either $S O(3)$ or as $S p(1)$, cf. 26, 27]

7. Obtain Nekrasov's partition function when the gauge group $S U(2)_{1} \times S U(2)_{2} \times$ $S U(2)_{3}$ couples to a half-hypermultiplet transforming in $\mathbf{2}_{1} \times \mathbf{2}_{2} \times \mathbf{2}_{3}$. This would be possible only in the $S p(1)$ formulation. Then compare the result with the appropriate conformal blocks.

8. Compactification on $S^{4}$ only gave us $b=1$. Find manifolds which give $b \neq 1$.

9. We used Nekrasov's partition function to associate a number to a gauge theory. We can also associate a number by taking the partition function of the topologically-twisted gauge theory on K3 or other four-manifolds, i.e. by considering the Donaldoson invariants. We would naively expect to find a different 2d CFT for each four-manifold. What are they?

10. Understand why we found Liouville theory, and why $\epsilon_{1}: \epsilon_{2}=b: 1 / b$. Our observation should have a place in the web of string dualities.

11. We interpreted the insertion of one Liouville energy-momentum tensor $T(z)$ as giving the Seiberg-Witten curve. What does multiple insertions of $T(z)$ correspond to? We identified the correlators of Liouville primaries as the integral of Nekrasov's partition function. What does the correlator of descendants correspond to?

12. Mathematically prove the equivalence of the square of the Seiberg-Witten differential and the semi-classical limit of $\langle T(z)\rangle$. Combined with the proof of the equivalence of the instanton partition function and the conformal blocks, this will provide a microscopic derivation of the Seiberg-Witten curve for quiver theories of $S U(2)$ gauge groups. cf. 28 . 
13. It is natural in the framework of [4] to insert Wilson loops on the gauge theory side. What does it correspond to on the Liouville theory?

14. On the Liouville theory side, there are ZZ branes and FZZT branes. What do they correspond to on the gauge theory side?

15. Both the Liouville theory and the supersymmetric gauge theory have been used to discuss geometric Langlands duality. Does our observation have anything to say about it?

16. Extend the whole of our analysis to the $A_{N-1}$ theory, i.e. when $N$, not just two, M5-branes are used (and to type $D$ or $E$ as well). The general structure of the instanton partition function appears to be compatible with the conformal blocks of W-algebras with a similar ADE classification. The dimension $k$ currents should map to the degree $k$ differentials in the canonical Seiberg-Witten curve. The main problem is to relate the spectrum of possible punctures to the spectrum of highest weight representations of the $\mathrm{W}$-algebras. The full partition function should be related to correlation functions of an ADE affine Toda theory, a variant of Liouville theory which has currents forming an ADE W-algebra.

Solutions to any of the problems listed above would be welcomed.

\section{Acknowledgments}

The authors have benefited from discussions with J. Maldacena, V. Pestun, N. Seiberg, C. Thorn and H. Verlinde. They also thank J. Teschner for informative correspondences. L.F.A. and D.G. are supported in part by the DOE grant DEFG02- 90ER40542. D.G. is supported in part by the Roger Dashen membership in the Institute for Advanced Study. YT is supported in part by the NSF grant PHY-0503584, and by the Marvin L. Goldberger membership at the Institute for Advanced Study.

\section{A. Liouville theory}

Here rudimentary facts of the Liouville theory are collected. More details can be found in the reviews [29, 30].

\section{A.1 Conformal blocks}

Conformal blocks for the Liouville theory are just the ones of the Virasoro algebra. These can be computed by the sewing procedure [31, 32, 33, 34]. The basis for this procedure is the following schematic expression 


$$
\left\langle\mathcal{O}_{1} \cdots \mathcal{O}_{k} \mathcal{O}_{k+1} \cdots \mathcal{O}_{n}\right\rangle=\sum_{i, \mathbf{m}, \mathbf{n}}\left\langle\mathcal{O}_{1} \cdots \mathcal{O}_{k} \mathcal{L}_{-\mathbf{m}} \phi_{i}\right\rangle_{M} K_{M N}^{-1}\left\langle\mathcal{L}_{-\mathbf{n}} \phi_{i} \mathcal{O}_{k+1} \cdots \mathcal{O}_{n}\right\rangle_{N}
$$

which allows to compute conformal blocks of a certain order in terms of lower order conformal blocks. The index $i$ runs over the primary fields and $\mathbf{m}, \mathbf{n}$ run over the descendants of such primary fields, namely

$$
\mathcal{L}_{-\mathbf{n}} \phi_{i}=L_{-n_{1}} L_{-n_{2}} \cdots L_{-n_{N}} \phi_{i}
$$

where $L_{n}$ are the generators of the Virasoro algebra with central charge $c$, and $k=$ $\sum_{i=1}^{N} n_{i}$ is the level of the descendant. The matrix $K$ is the so called Gram matrix, whose determinant is the Kac determinant. At level $k$, the indexes $M, N$ run over the partitions of $k$, and $K_{M N}$ are given by the inner product of the corresponding descendants of the primary field under consideration. For instance, at level two we get

$$
K=\left(\begin{array}{l}
\left\langle h\left|L_{2} L_{-2}\right| h\right\rangle\left\langle h\left|L_{1}^{2} L_{-2}\right| h\right\rangle \\
\left\langle h\left|L_{2} L_{-1}^{2}\right| h\right\rangle\left\langle h\left|L_{1}^{2} L_{-1}^{2}\right| h\right\rangle
\end{array}\right)=\left(\begin{array}{cc}
4 h+c / 2 & 6 h \\
6 h & 4 h(1+2 h)
\end{array}\right)
$$

where $|h\rangle$ is a primary of dimension $h$. It is then possible to express the conformal blocks we use in this paper by sewing elementary building blocks, of the form

$$
\begin{array}{r}
R_{M}\left(h_{1}, h_{2}, h_{3}\right)=\frac{\left\langle\mathcal{O}_{1} \mathcal{O}_{2} \mathcal{L}_{-\mathbf{m}} \phi_{3}\right\rangle}{\left\langle\mathcal{O}_{1} \mathcal{O}_{2} \phi_{3}\right\rangle} \\
S_{M, N}\left(h_{1}, h_{2}, h_{3}\right)=\frac{\left\langle\mathcal{L}_{-\mathbf{m}} \phi_{1} \mathcal{O}_{2} \mathcal{L}_{-\mathbf{n}} \phi_{3}\right\rangle}{\left\langle\phi_{1} \mathcal{O}_{2} \phi_{3}\right\rangle}
\end{array}
$$

We can represent pictorially $R$ and $S$ as stars with three legs. The difference between the two is the amount of external legs, two for the former and one for the later. $K^{-1}$ then represents a propagator, which joins internal legs, see Figure $⿴$.

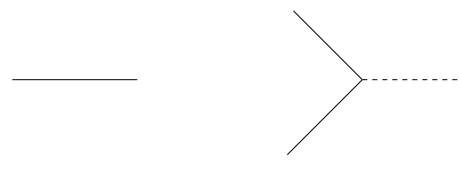

(a) (b)

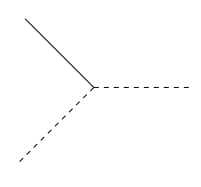

(c)

Figure 4: Pictorial representation of the propagator $K^{-1}$, shown in (a), the vertex with two external legs $R$, shown in (b) and the vertex with one external leg $S$, shown in (c). External legs are represented with solid lines, while internal legs are represented with dashed lines.

$R$ and $S$ depend on the dimensions of the primaries under consideration, while $K$ depends on the dimension of the primary interchanged and on the central charge. 
Higher order conformal blocks can then be computed by sewing this building blocks, for instance, the four-point conformal block on the sphere is

$$
R\left(h_{4}, h_{3}, h\right) K^{-1}(h) R\left(h, h_{2}, h_{1}\right)
$$

while that for five points is

$$
R\left(h_{4}, h_{5}, h_{b}\right) K^{-1}\left(h_{b}\right) S\left(h_{b}, h_{3}, h_{a}\right) K^{-1}\left(h_{a}\right) R\left(h_{a}, h_{2}, h_{1}\right)
$$

and so on, while the one point on the torus is given by

$$
\operatorname{Tr}\left(K^{-1}(h) S\left(h, h_{1}, h\right)\right)
$$

the two-point function is

$$
\operatorname{Tr}\left(K^{-1}\left(h_{a}\right) S\left(h_{a}, h_{2}, h_{b}\right) K^{-1}\left(h_{b}\right) S\left(h_{b}, h_{1}, h_{a}\right)\right)
$$

and so on, see Figure 5.

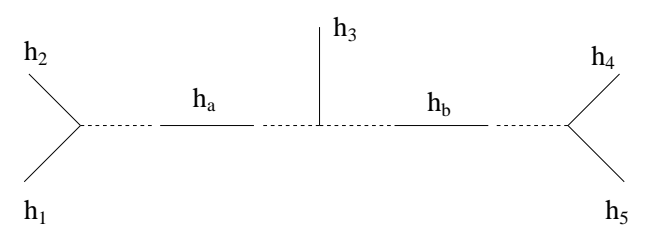

(a)

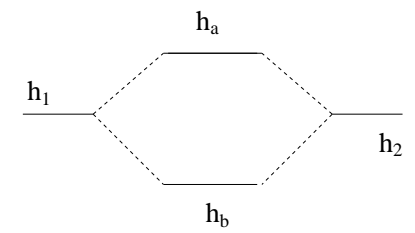

(b)

Figure 5: Sewing of building blocks into sphere (a) and and torus (b) conformal blocks. In the figure we see a five-point conformal block in the sphere and two points on the torus.

The level of each contribution is then fixed by the level of its internal propagators, $k_{1}, k_{2}, \ldots, k_{n}$. The full conformal block is obtained by multiplying each contribution by $q_{1}^{k_{1}} \cdots q_{n}^{k_{n}}$ and adding up all contributions. For instance, for the five-point conformal block on the sphere and the two-point conformal block on the torus we obtain

$$
\begin{aligned}
\mathcal{F}_{g=0}^{5 p t} & =1+\frac{\left(-h_{1}+h_{2}+h_{a}\right)\left(h_{3}+h_{a}-h_{b}\right)}{2 h_{a}} q_{1}+\frac{\left(-h_{4}+h_{5}+h_{b}\right)\left(h_{3}-h_{a}+h_{b}\right)}{2 h_{b}} q_{2}+\cdots \\
\mathcal{F}_{g=1}^{2 p t} & =1+\frac{\left(h_{1}+h_{a}-h_{b}\right)\left(h_{2}+h_{a}-h_{b}\right)}{2 h_{a}} q_{1}+\frac{\left(h_{1}-h_{a}+h_{b}\right)\left(h_{2}-h_{a}+h_{b}\right)}{2 h_{b}} q_{2}+\cdots
\end{aligned}
$$

In section five we were interested in computing conformal blocks with an insertion of the energy momentum tensor $T(z)$. These can be easily computed by considering modified vertices, analogous to the ones defined above, which take into account the appropriate $T(z)$ insertion, for instance

$$
R \rightarrow R T=\frac{\left\langle\mathcal{O}_{1} \mathcal{O}_{2} T(z) \mathcal{L}_{-\mathbf{m}} \phi_{3}\right\rangle}{\left\langle\mathcal{O}_{1} \mathcal{O}_{2} \phi_{3}\right\rangle}
$$


and so on, where $T(z)=\sum L_{n} z^{-n-2}$. Note that after inserting $T(z)$ even the conformal block at level zero is non trivial.

\section{A.2 The DOZZ formula}

Let us first define Barnes' double Gamma function 35 which is ubiquitous in our discussion of the Liouville theory and Nekrasov's partition function. Barnes' double zeta function is

$$
\zeta_{2}\left(s ; x \mid \epsilon_{1}, \epsilon_{2}\right)=\sum_{m, n}\left(m \epsilon_{1}+n \epsilon_{2}+x\right)^{-s}=\frac{1}{\Gamma(s)} \int_{0}^{\infty} \frac{d t}{t} t^{s} \frac{e^{-t x}}{\left(1-e^{-\epsilon_{1} t}\right)\left(1-e^{-\epsilon_{2} t}\right)} .
$$

This is the logarithm of Barnes' double-Gamma function,

$$
\Gamma_{2}\left(x \mid \epsilon_{1}, \epsilon_{2}\right)=\left.\exp \frac{d}{d s}\right|_{0} \zeta_{2}\left(s, x \mid \epsilon_{1}, \epsilon_{2}\right) .
$$

The arguments $\epsilon_{1,2}$ in $\Gamma_{2}$ will be often omitted if there is no confusion.

Assume $\epsilon_{1,2} \in \mathbb{R}_{>0}$. Then Barnes' double-Gamma function is analytic in $x$ except at the poles at $x=-\left(m \epsilon_{1}+n \epsilon_{2}\right)$ where $(m, n)$ is a pair of non-negative integers. Therefore one can think of Barnes' double-Gamma as the regularized infinite product

$$
\Gamma_{2}\left(x \mid \epsilon_{1}, \epsilon_{2}\right) \propto \prod_{m, n \geq 0}\left(x+m \epsilon_{1}+n \epsilon_{2}\right)^{-1} .
$$

Furthermore it is real when $x$ is real. As such,

$$
\Gamma_{2}\left(x^{*}\right)=\Gamma_{2}(x)^{*} .
$$

Another relation we need is

$$
\Gamma_{2}\left(x+\epsilon_{1}\right) \Gamma_{2}\left(x+\epsilon_{2}\right)=x \Gamma_{2}(x) \Gamma_{2}\left(x+\epsilon_{1}+\epsilon_{2}\right) .
$$

This is a natural property the infinite product in the right hand side of (A.12) would have.

We will also need the infinite product expansion when $\epsilon_{1}>0, \epsilon_{2}<0$, which is given by

$$
\Gamma_{2}\left(x \mid \epsilon_{1}, \epsilon_{2}\right) \propto \prod_{m, n \geq 1}\left(x+(m-1) \epsilon_{1}-n \epsilon_{2}\right)^{+1} .
$$

Note that we have zeros instead of poles in this case.

The Liouville theory has the parameter $b$. The central chrage is then

$$
c=1+6 Q^{2}, \quad Q=b+1 / b .
$$


The three-point function is given by the DOZZ formula [5, 6, 7, 29, 14]

$$
\begin{aligned}
& \left\langle V_{\alpha_{1}}\left(z_{1}\right) V_{\alpha_{2}}\left(z_{2}\right) V_{\alpha_{3}}\left(z_{3}\right)\right\rangle \\
& \quad=\left|z_{12}\right|^{2\left(\Delta_{1}+\Delta_{2}-\Delta_{3}\right)}\left|z_{23}\right|^{2\left(\Delta_{2}+\Delta_{3}-\Delta_{1}\right)}\left|z_{31}\right|^{2\left(\Delta_{3}+\Delta_{1}-\Delta_{2}\right)} C\left(\alpha_{1}, \alpha_{2}, \alpha_{3}\right)
\end{aligned}
$$

where $\Delta_{i}$ is the dimension of the operators $V_{\alpha_{i}}=e^{2 \alpha \phi}$ given by

$$
\Delta_{i}=\alpha_{i}\left(Q-\alpha_{i}\right)
$$

and

$$
\begin{aligned}
& C\left(\alpha_{1}, \alpha_{2}, \alpha_{3}\right)=\left[\pi \mu \gamma\left(b^{2}\right) b^{2-2 b^{2}}\right]^{\left(Q-\alpha_{1}-\alpha_{2}-\alpha_{3}\right) / b} \\
& \times \frac{\Upsilon^{\prime}(0) \Upsilon\left(2 \alpha_{1}\right) \Upsilon\left(2 \alpha_{2}\right) \Upsilon\left(2 \alpha_{3}\right)}{\Upsilon\left(\alpha_{1}+\alpha_{2}+\alpha_{3}-Q\right) \Upsilon\left(\alpha_{1}+\alpha_{2}-\alpha_{3}\right) \Upsilon\left(\alpha_{1}-\alpha_{2}+\alpha_{3}\right) \Upsilon\left(-\alpha_{1}+\alpha_{2}+\alpha_{3}\right)}
\end{aligned}
$$

where

$$
\Upsilon(x)=\frac{1}{\Gamma_{2}\left(x \mid b, b^{-1}\right) \Gamma_{2}\left(Q-x \mid b, b^{-1}\right)} .
$$

and

$$
\gamma(x)=\Gamma(x) / \Gamma(1-x) .
$$

\section{B. Nekrasov formulae}

Here we provide the precise formulae of Nekrasov's partition function [2, 3] for the $U(2)$ quiver theories we discussed in the main part of the paper.

\section{B.1 Instanton part}

The instanton partition function is computed by performing the path integral by localizing with respect to the $S O(4)$ rotation specified by $\left(\epsilon_{1}, \epsilon_{2}\right)$. We set $\epsilon_{+}=\epsilon_{1}+\epsilon_{2}$; this was identified with $Q$ in the main part of the text. Localization fixes the gauge field configuration to be instantons sitting at the origin; for each $U(2)$ gauge group such fixed instantons are labeled by a pair of Young tableaux $\vec{Y}=\left(Y_{1}, Y_{2}\right)$, and the instanton number is given by the total number of boxes $|\vec{Y}|=\left|Y_{1}\right|+\left|Y_{2}\right|$. Then the contribution is weighted by the instanton factor $q^{|\vec{Y}|}$ where

$$
q=\exp \left(2 \pi i \tau_{\mathrm{UV}}\right), \quad \tau_{\mathrm{UV}}=\frac{4 \pi i}{g_{\mathrm{UV}}^{2}}+\frac{\theta_{\mathrm{UV}}}{2 \pi} .
$$

Note that the coupling constant receives finite renormalization, even though the conformal quivers are finite theories; therefore it is important to keep in mind that $\tau$ appearing in Nekrasov's partition function is the UV coupling in a particular renormalization scheme. We have seen that this scheme is a particularly natural one in the main part of the paper. 


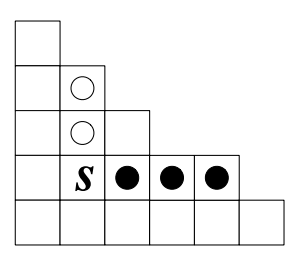

Figure 6: Definition of the arm-length and the leg-length. For a box $s$ in a Young tableau displayed above, the leg-length is the number of boxes to the right of $s$, marked by black disks, and the arm-length is the number of boxes on top of $s$.

For a linear quiver with $N U(2)$ gauge groups, the partition function is then

$$
\begin{aligned}
Z_{\text {inst }} & =\sum_{\vec{Y}_{1}, \vec{Y}_{2}, \ldots, \vec{Y}_{N}}\left(\prod_{i=1}^{N} q_{i}^{\left|\vec{Y}_{i}\right|} z_{\text {vector }}\left(\vec{a}_{i}, \vec{Y}_{i}\right)\right) z_{\text {antifund }}\left(\vec{a}_{1}, \vec{Y}_{1}, \mu_{1}\right) z_{\text {antifund }}\left(\vec{a}_{1}, \vec{Y}_{1}, \mu_{2}\right) \\
& \times\left(\prod_{i=1}^{N-1} z_{\text {bifund }}\left(\vec{a}_{i}, \vec{Y}_{i} ; \vec{a}_{i+1}, \vec{Y}_{i+1} ; m_{i}\right)\right) z_{\text {fund }}\left(\vec{a}_{N}, \vec{Y}_{N}, \mu_{3}\right) z_{\text {fund }}\left(\vec{a}_{N}, \vec{Y}_{N}, \mu_{4}\right) .
\end{aligned}
$$

Here $\vec{a}_{i}=\left(a_{i, 1}, a_{i, 2}\right)$ is the diagonal of the adjoint scalar, $\vec{Y}_{i}$ the pair of the Young tableaux specifying the fixed instanton, $q_{i}$ is the exponentiated UV gauge coupling of the $i$-th $S U(2)$ gauge group. $m_{i}$ is the mass of the bifundamental hypermultiplet charged under $S U(2)_{i}$ and $S U(2)_{i+1} . \quad \mu_{1,2,3,4}$ are the masses of the fundamentals. $z_{\text {vector }}, z_{\text {bifund }}$ etc. are the contribution of the vector multiplet, the bifundamental hypermultiplet, etc. defined below. For a necklace quiver one needs to replace four fundamentals by one bifundamental charged under $S U(2)_{n}$ and $S U(2)_{1}$. For the $\mathcal{N}=$ $2^{*} S U(2)$ theory one needs to put the contribution from the adjoint hypermultiplet $z_{\text {adj }}$.

Let $Y=\left(\lambda_{1} \geq \lambda_{2} \geq \cdots\right)$ be a Young tableau where $\lambda_{i}$ is the height of the $i$-th column. We set $\lambda_{i}=0$ when $i$ is larger than the width of the tableau. Let $Y^{T}=\left(\lambda_{1}^{\prime} \geq \lambda_{2} \geq \cdots\right)$ be its transpose. For a box $s$ at the coordinate $(i, j)$, we let its arm-length $A_{Y}(s)$ and leg-length $L_{Y}(s)$ with respect to the tableau $Y$ to be

$$
A_{Y}(s)=\lambda_{i}-j, \quad L_{Y}(s)=\lambda_{j}^{\prime}-i,
$$

see Fig. 6. Note that they can be negative when the box $s$ is outside the tableau. We then define a function $E$ by

$$
E\left(a, Y_{1}, Y_{2}, s\right)=a-\epsilon_{1} L_{Y_{2}}(s)+\epsilon_{2}\left(A_{Y_{1}}(s)+1\right) .
$$

We use the vector symbol $\vec{a}$ to stand for pairs $\vec{a}=\left(a_{1}, a_{2}\right)$, e.g. $\vec{a}_{1}=\left(a_{1,1}, a_{2,1}\right)$, $\vec{Y}=\left(Y_{1}, Y_{2}\right)$, etc. We can now define the contribution of a bifundamental [36, 28]:

$$
\begin{aligned}
& z_{\mathrm{bifund}}(\vec{a}, \vec{Y} ; \vec{b}, \vec{W} ; m)= \\
& \quad \prod_{i, j=1}^{2} \prod_{s \in Y_{i}}\left(E\left(a_{i}-b_{j}, Y_{i}, W_{j}, s\right)-m\right) \prod_{t \in W_{j}}\left(\epsilon_{+}-E\left(b_{j}-a_{i}, W_{j}, Y_{i}, t\right)-m\right)
\end{aligned}
$$


Note that this is not symmetric under the exchange between $(\vec{a}, \vec{Y})$ and $(\vec{b}, \vec{W})$; instead it satisfies

$$
z_{\text {bifund }}(\vec{a}, \vec{Y} ; \vec{b}, \vec{W} ; m)=z_{\text {bifund }}\left(\vec{b}, \vec{W} ; \vec{a}, \vec{Y} ; \epsilon_{+}-m\right)
$$

This behavior comes from a subtlety in Nekrasov's setup: the gauge group is strictly speaking $U(2) \times U(2)$ instead of $S U(2) \times S U(2)$, so the representations $\mathbf{2} \otimes \overline{\mathbf{2}}$ and $\overline{\mathbf{2}} \otimes \mathbf{2}$ are different. The formula above means that by exchanging a bifundamental with a anti-bifundamental, we need to flip the mass as $m \rightarrow \epsilon_{+}-m$.

The contribution of an adjoint hypermultiplet is now easy to define. It is

$$
z_{\text {adj }}(\vec{a}, \vec{Y}, m)=z_{\text {bifund }}(\vec{a}, \vec{Y}, \vec{a}, \vec{Y}, m)
$$

Then the contribution of a vector multiplet is

$$
z_{\text {vector }}(\vec{a}, \vec{Y})=1 / z_{\text {adj }}(\vec{a}, \vec{Y}, 0)
$$

The contribution from (anti)fundamental hypermultiplets is defined as follows:

$$
\begin{aligned}
z_{\text {fund }}(\vec{a}, \vec{Y}, m) & =\prod_{i=1}^{2} \prod_{s \in Y_{i}}\left(\phi\left(a_{i}, s\right)-m+\epsilon_{+}\right), \\
z_{\text {antifund }}(\vec{a}, \vec{Y}, m) & =z_{\text {fund }}\left(\vec{a}, \vec{Y}, \epsilon_{+}-m\right)
\end{aligned}
$$

where $\phi(a, s)$ for the box $s=(i, j)$ is defined as

$$
\phi(a, s)=a+\epsilon_{1}(i-1)+\epsilon_{2}(j-1) .
$$

$z_{(\text {anti)fund }}$ and $z_{\text {bifund }}$ satisfies two important relations:

$$
\begin{aligned}
& z_{\text {bifund }}(\vec{a}, \vec{Y}, \vec{\mu}, \emptyset, m)=z_{\text {fund }}(\vec{a}, \vec{Y}, m+\mu) z_{\text {fund }}(\vec{a}, \vec{Y}, m-\mu), \\
& z_{\text {bifund }}(\vec{\mu}, \emptyset, \vec{a}, \vec{Y}, m)=z_{\text {antifund }}(\vec{a}, \vec{Y}, m+\mu) z_{\text {antifund }}(\vec{a}, \vec{Y}, m-\mu)
\end{aligned}
$$

where $\vec{\mu}=\left(\mu_{1}, \mu_{2}\right)=(\mu,-\mu)$, and $\emptyset$ stands for a pair of empty Young tableaux. The relation $(\mathbb{B} .12)$ means that a bifundamental, with the second $S U(2)$ at zero coupling, behaves as two fundamental hypermultiplets of the first $S U(2)$ with mass $m \pm \mu$ where $(\mu,-\mu)$ are the diagonal entries of the adjoint scalar of the second $S U(2)$; this is as expected. The relation (B.13) can be understood similarly.

\section{B.2 One-loop part}

The partition function discussed above contains only the contribution from the instantons. The full partition function also contains the classical and the one-loop part, i.e.

$$
Z_{\text {Nekrasov }}=Z_{\text {classical }} Z_{1-\text { loop }} Z_{\text {inst }}
$$


The prepotential $F$ can then be recovered from its logarithm,

$$
F\left(\tau_{i} ; m_{a} ; a_{i}\right)=\lim _{\epsilon_{1}, \epsilon_{2} \rightarrow 0} \epsilon_{1} \epsilon_{2} \log Z_{\text {Nekrasov }}\left(\tau_{i} ; m_{i} ; a_{i}\right) .
$$

The classical part is simply

$$
Z_{\text {classical }}=\exp \left[-\frac{1}{\epsilon_{1} \epsilon_{2}} \sum_{i}(2 \pi i) \tau_{i} a_{i}^{2}\right] .
$$

The basic ingredient of the one-loop part is the logarithm of Barnes' double gamma functions:

$$
\gamma_{\epsilon_{1}, \epsilon_{2}}(x)=\log \Gamma_{2}\left(x+\epsilon_{+} \mid \epsilon_{1}, \epsilon_{2}\right) .
$$

When $\hbar=\epsilon_{1}=-\epsilon_{2}, \gamma_{\hbar,-\hbar}(x)$ has the expansion

$$
\gamma_{\hbar,-\hbar}(x)=\hbar^{-2}\left(\frac{1}{2} x^{2} \log x-\frac{3}{4} x^{2}\right)-\frac{1}{12} \log x+\sum_{g=2}^{\infty} \frac{B_{2 g}}{2 g(2 g-2)}\left(\frac{\hbar}{x}\right)^{2 g-2}
$$

where $B_{2 g}$ is the Bernoulli number; one recognizes the limit

$$
\lim _{\hbar \rightarrow 0} \hbar^{2} \gamma_{\hbar,-\hbar}(x)=\frac{1}{2} x^{2} \log x-\frac{3}{4} x^{2}
$$

to be the standard one-loop contribution to the prepotential of a hypermultiplet of mass $x$, i.e. $\left[\left(x^{2} / 2\right) \log x-(3 / 4) x^{2}\right]^{\prime \prime}=\log x$.

Then, for a linear quiver with $N$ gauge groups, the one-loop part is given by combining this factor for all elementary particles:

$$
\begin{aligned}
Z_{1 \text {-loop }}= & \left(\prod_{i=1}^{N} z_{\text {vector }}^{1 \text {-loop }}\left(\vec{a}_{i}\right)\right) z_{\text {antifund }}^{1 \text {-loop }}\left(\vec{a}_{1}, \mu_{1}\right) z_{\text {antifund }}^{1 \text {-loop }}\left(\vec{a}_{1}, \mu_{2}\right) \\
& \times\left(\prod_{i=1}^{N-1} z_{\text {bifund }}^{1 \text {-loop }}\left(\vec{a}_{i}, \vec{a}_{i+1}, m_{i}\right)\right) z_{\text {fund }}^{1 \text { loop }}\left(\vec{a}_{N}, \mu_{3}\right) z_{\text {fund }}^{1 \text {-loop }}\left(\vec{a}_{N}, \mu_{4}\right)
\end{aligned}
$$

where

$$
\begin{aligned}
z_{\text {vector }}^{1-\text { loop }}(\vec{a}) & =\prod_{i<j} \exp \left[-\gamma_{\epsilon_{1}, \epsilon_{2}}\left(a_{i}-a_{j}-\epsilon_{1}\right)-\gamma_{\epsilon_{1}, \epsilon_{2}}\left(a_{i}-a_{j}-\epsilon_{2}\right)\right] \\
z_{\text {fund }}^{1 \text {-loop }}(\vec{a}, \mu) & =\prod_{i} \exp \left[\gamma_{\epsilon_{1}, \epsilon_{2}}\left(a_{i}-\mu\right)\right] \\
z_{\text {antifund }}^{1-\text { loop }}(\vec{a}, \mu) & =\prod_{i} \exp \left[\gamma_{\epsilon_{1}, \epsilon_{2}}\left(-a_{i}+\mu-\epsilon_{+}\right)\right] \\
z_{\text {bifund }}^{1 \text { l-oop }}(\vec{a}, \vec{b}, m) & =\prod_{i, j} \exp \left[\gamma_{\epsilon_{1}, \epsilon_{2}}\left(a_{i}-b_{j}-m\right)\right]
\end{aligned}
$$

The contribution of a $U(N)$ adjoint hypermultiplet is given by $z_{\text {bifund }}^{1-\text { loop }}(\vec{a}, \vec{a}, m)$. 
The one-loop factor for the vector multiplet (B.21) is different from the one in [3], in that theirs does not have the shift by $-\epsilon_{1,2}$ in it. A more detailed analysis [11, 12] showed that (B.21) is more appropriate. Using Eq.(128) of [11] or using Eq.(3) of [12], one finds that the contribution of a five-dimensional vector multiplet with mass $\mu$, which has $j_{R}=1 / 2$ in their notation, is given by

$$
\prod_{m, n=1}^{\infty}\left(1-e^{-(m-1) \epsilon_{1}+n \epsilon_{2}-\mu}\right)^{-1}\left(1-e^{-m \epsilon_{1}+(n-1) \epsilon_{2}-\mu}\right)^{-1} .
$$

Taking the four-dimensional limit involves replacing $1-e^{-x}$ by $x$ in the infinite product. Here we need to remind ourselves $\epsilon_{1}>0$ and $\epsilon_{2}<0 .{ }^{6}$ Using (A.15), we find that it becomes

$$
\Gamma_{2}\left(\mu \mid \epsilon_{1}, \epsilon_{2}\right)^{-1} \Gamma_{2}\left(\mu+\epsilon_{1}+\epsilon_{2} \mid \epsilon_{1}, \epsilon_{2}\right)^{-1}
$$

which reproduces (B.21), up to one factor of $\mu$.

Similarly, using the same starting point but with $j_{R}=0$ which corresponds to a hypermultiplet, one finds

$$
\prod_{m, n=1}^{\infty}\left(1-e^{-(m-1 / 2) \epsilon_{1}+(n-1 / 2) \epsilon_{2}-\mu}\right)
$$

which in the four-dimensional limit becomes

$$
\Gamma_{2}\left(\mu+\frac{\epsilon_{+}}{2} \mid \epsilon_{1}, \epsilon_{2}\right)
$$

Again, we used (A.15). This reproduces (B.22) with the shift of $\mu$ by $\epsilon_{+} / 2$. It just reflects the difference in the conventions in [3] and in [11, 12] of the zero of the hypermultiplet mass.

\section{B.3 Example}

As an example we present here a result for $S U(2)$ theory with $N_{f}=4$ massless flavors. Performing the instanton sum and combining with the one-loop and the classical parts, we obtain the low-energy prepotential:

$$
\begin{aligned}
& (2 \pi i) \tau_{\mathrm{IR}} a^{2}= \\
& \quad(2 \pi i) \tau_{\mathrm{UV}} a^{2}-(\log 16) a^{2}+a^{2}\left(\frac{1}{2} q+\frac{13}{64} q^{2}+\frac{23}{192} q^{3}+\frac{2701}{32768} q^{4}+\cdots\right)
\end{aligned}
$$

where $q=\exp \left(2 \pi i \tau_{\mathrm{UV}}\right)$. The first, the second, and the third terms come from the classical, the one-loop and the instanton contributions, respectively. Let us define

$$
q_{\mathrm{IR}}=\exp \left(4 \pi i \tau_{\mathrm{IR}}\right), \quad \tau_{\mathrm{IR}}=\frac{4 \pi i}{g_{\mathrm{IR}}^{2}}+\frac{\theta_{\mathrm{IR}}}{2 \pi} .
$$

\footnotetext{
${ }^{6}$ The authors thank T. Okuda for pointing the error they made in v1.
} 


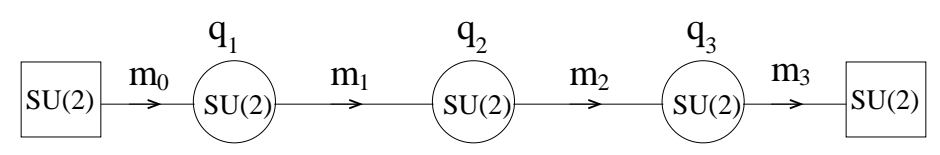

Figure 7: Quiver theory corresponding to the sphere with six punctures.

Note that in the presence of the massless hypermultiplets in the doublet representation there is a shift symmetry of the theta angle, $\theta \rightarrow \theta+\pi \cdot q_{\mathrm{IR}}$ is designed to be invariant under this shift.

Inverting (B.29), we find

$$
q_{\mathrm{UV}}=16 q_{\mathrm{IR}}^{1 / 2}-128 q_{\mathrm{IR}}+704 q_{\mathrm{IR}}^{3 / 2}-3072 q_{\mathrm{IR}}^{2}+\cdots=\frac{\theta_{2}\left(q_{\mathrm{IR}}\right)^{4}}{\theta_{3}\left(q_{\mathrm{IR}}\right)^{4}}
$$

or equivalently $q_{\mathrm{UV}}=\lambda\left(2 \tau_{\mathrm{IR}}\right)$ where $\lambda$ is the modular lambda function. This means that the double cover of a sphere with four branch points with cross ratio $q_{\mathrm{UV}}$ is an elliptic curve with modulus $2 \tau_{\mathrm{IR}}$. This relation was first noticed in 10.

\section{C. $U(1)$ factors}

According to our conjecture, Nekrasov's instanton partition function on certain generalized quiver theories coincides with the conformal blocks on the corresponding two dimensional Riemann surface, upon stripping off a " $U(1)$ factor" which presumably arises from the fact that the Nekrasov partition function is computed for $U(2)$ groups, rather than $S U(2)$. In this appendix we write down the explicit form of such factors for the case of the sphere and the torus.

\section{C.1 Sphere}

The quiver gauge theory corresponding to the sphere with $n+3$ punctures has $\prod_{i}^{n} S U(2)_{i}$ gauge group, as shown in Figure [7, whose instanton numbers are counted by powers of $q_{i}$. In addition we have bi-fundamental matter with masses $m_{i}, i=$ $0, \ldots, n$, and $m_{0} \equiv m_{n+1}$. The explicit result for the first few cases is

$$
\begin{aligned}
Z_{U(1)}^{g=0, n=4}= & (1-q)^{2 m_{0}\left(Q-m_{1}\right)} \\
Z_{U(1)}^{g=0, n=5}= & \left(1-q_{1}\right)^{2 m_{0}\left(Q-m_{1}\right)}\left(1-q_{2}\right)^{2 m_{1}\left(Q-m_{2}\right)}\left(1-q_{1} q_{2}\right)^{2 m_{0}\left(Q-m_{2}\right)} \\
Z_{U(1)}^{g=0, n=6}= & \left(1-q_{1}\right)^{2 m_{0}\left(Q-m_{1}\right)}\left(1-q_{2}\right)^{2 m_{1}\left(Q-m_{2}\right)}\left(1-q_{3}\right)^{2 m_{2}\left(Q-m_{3}\right)} \times \\
\times & \left(1-q_{1} q_{2}\right)^{2 m_{0}\left(Q-m_{2}\right)}\left(1-q_{2} q_{3}\right)^{2 m_{1}\left(Q-m_{3}\right)}\left(1-q_{1} q_{2} q_{3}\right)^{2 m_{0}\left(Q-m_{3}\right)}
\end{aligned}
$$

Note that the general pattern is easily recognizable. Given a set of consecutive nodes, let's say $q_{1}, \ldots, q_{m}$, there is a factor of the form

$$
\prod_{\ell=0}^{\infty}\left(1-q_{1} \cdots q_{m}\right)^{2 m_{\text {in }}\left(Q-m_{\mathrm{out}}\right)}
$$




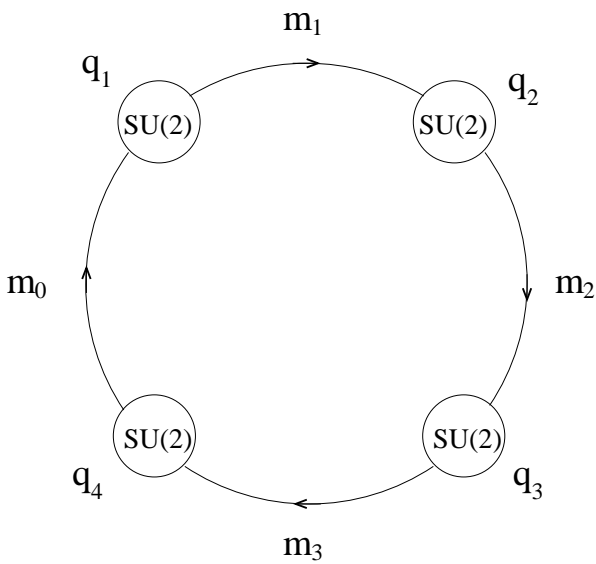

Figure 8: Necklace quiver theory corresponding to the torus with four punctures.

where $m_{\text {in }}$ is the mass of the adjoint bifundamental that enters into the set of nodes and $m_{\text {out }}$ is the mass of the adjoint bifundamental that exits the set of nodes. One then has to multiply the contributions from all such sets. We checked this general pattern up to $n=7$.

Note that when defining $m_{\text {in }}$ and $m_{\text {out }}$, we have assigned an orientation for the bifundamental matter, shown by the direction of the arrows in figure 7. This of course wouldn't make sense when considering $S U(2)$ gauge groups, but remember that Nekrasov's partition function was computed for $U(2)$ quiver gauge theories.

\section{C.2 Torus}

In the case of the torus, we are led to consider the instanton partition function of necklace quiver theories as the one shown in Figure 8. These necklace theories have $\prod_{i}^{n} S U(2)_{i}$ gauge group, as shown in the figure, whose instanton numbers are counted by powers of $q_{i}$. In addition, we have bi-fundamental matter with mass $m_{0} \equiv m_{n}$, $m_{1}$, etc. Furthermore, when computing the instanton partition function we need to choose an orientation for the bi-fundamental matter, as explained above.

The explicit result for the first few cases is

$$
\begin{aligned}
Z_{U(1)}^{g=1, n=1}= & \prod_{\ell=0}^{\infty}\left(1-q_{1}^{\ell+1}\right)^{2 m_{1}\left(Q-m_{1}\right)-1} \\
Z_{U(1)}^{g=1, n=2}= & \prod_{\ell=0}^{\infty}\left(1-q_{1}^{\ell+1} q_{2}^{\ell+1}\right)^{2 m_{1}\left(Q-m_{1}\right)+2 m_{2}\left(Q-m_{2}\right)-1} \times \\
\times \quad & \left(1-q_{1}^{\ell+1} q_{2}^{\ell}\right)^{2 m_{2}\left(Q-m_{1}\right)}\left(1-q_{1}^{\ell} q_{2}^{\ell+1}\right)^{2 m_{1}\left(Q-m_{2}\right)} \\
Z_{U(1)}^{g=1, n=3}= & \prod_{\ell=0}^{\infty}\left(1-q_{1}^{\ell+1} q_{2}^{\ell+1} q_{3}^{\ell+1}\right)^{2 m_{1}\left(Q-m_{1}\right)+2 m_{2}\left(Q-m_{2}\right)+2 m_{3}\left(Q-m_{3}\right)-1} \times \\
\times \quad & \left(1-q_{1}^{\ell+1} q_{2}^{\ell+1} q_{3}^{\ell}\right)^{2 m_{3}\left(Q-m_{2}\right)}\left(1-q_{1}^{\ell+1} q_{2}^{\ell} q_{3}^{\ell+1}\right)^{2 m_{2}\left(Q-m_{1}\right)}\left(1-q_{1}^{\ell} q_{2}^{\ell+1} q_{3}^{\ell+1}\right)^{2 m_{1}\left(Q-m_{3}\right)} \times \\
\times \quad & \left(1-q_{1}^{\ell+1} q_{2}^{\ell} q_{3}^{\ell}\right)^{2 m_{3}\left(Q-m_{1}\right)}\left(1-q_{1}^{\ell} q_{2}^{\ell+1} q_{3}^{\ell}\right)^{2 m_{1}\left(Q-m_{2}\right)}\left(1-q_{1}^{\ell} q_{2}^{\ell} q_{3}^{\ell+1}\right)^{2 m_{2}\left(Q-m_{3}\right)} \quad \text { (C.7) }
\end{aligned}
$$


The general pattern is easy to recognize. First of all, given the quiver diagram with $n$-nodes, there is a factor

$$
\prod_{\ell=0}^{\infty}\left(1-q_{1}^{\ell+1} \cdots q_{n}^{\ell+1}\right)^{\sum_{i=1}^{n} 2 m_{i}\left(Q-m_{i}\right)-1}
$$

Second, given a set of consecutive nodes, let's say $q_{1}, \ldots, q_{m}$, there is a factor of the form

$$
\prod_{\ell=0}^{\infty}\left(1-q_{1}^{\ell+1} \cdots q_{m}^{\ell+1} q_{m+1}^{\ell} \cdots q_{n}^{\ell}\right)^{2 m_{\mathrm{in}}\left(Q-m_{\mathrm{out}}\right)},
$$

where $m_{\text {in }}$ is the mass of the adjoint bifundamental that enters into the set of nodes and $m_{\text {out }}$ is the mass of the adjoint bifundamental that exits the set of nodes. Another interpretation is to consider all possible paths on the quiver starting on a node, say $q_{i}$, and go $k$ steps along the arrow, winding many times on the circle. Then we can easily see that the expressions written above equals

$$
Z_{U(1)}^{g=1}=\frac{\prod_{i=1}^{n} \prod_{k=0}^{\infty}\left(1-q_{i} q_{i+1} \cdots q_{i+k}\right)^{2 m_{i-1}\left(Q-m_{i+k}\right)}}{\prod_{\ell=1}^{\infty}\left(1-q_{1}^{\ell} q_{2}^{\ell} \cdots q_{n}^{\ell}\right)}
$$

where the subscripts of $q_{i}$ and $m_{i}$ are considered modulo $n$. We checked this general pattern up to $n=4$.

As a further check, note that if we disconnect one of the nodes, sending $q_{n} \rightarrow 0$, we reobtain the $U(1)$ factors of the linear quiver (remembering that $m_{n}$ and $m_{0}$ are identified.)

\section{References}

[1] D. Gaiotto, "N $=2$ dualities," arXiv:0904.2715 [hep-th].

[2] N. A. Nekrasov, "Seiberg-Witten Prepotential From Instanton Counting," Adv. Theor. Math. Phys. 7 (2004) 831-864, arXiv:hep-th/0206161.

[3] N. Nekrasov and A. Okounkov, "Seiberg-Witten theory and random partitions," arXiv:hep-th/0306238.

[4] V. Pestun, "Localization of gauge theory on a four-sphere and supersymmetric Wilson loops," arXiv:0712.2824 [hep-th].

[5] H. Dorn and H.-J. Otto, "Two and three-point functions in Liouville theory," Nucl. Phys. B429 (1994) 375-388, arXiv:hep-th/9403141.

[6] A. B. Zamolodchikov and A. B. Zamolodchikov, "Structure constants and conformal bootstrap in Liouville field theory," Nucl. Phys. B477 (1996) 577-605,

arXiv:hep-th/9506136. 
[7] J. Teschner, "On the Liouville three point function," Phys. Lett. B363 (1995) 65-70, arXiv:hep-th/9507109.

[8] N. Seiberg and E. Witten, "Monopoles, duality and chiral symmetry breaking in $\mathcal{N}=2$ supersymmetric QCD," Nucl. Phys. B431 (1994) 484-550, arXiv:hep-th/9408099.

[9] N. Dorey, V. V. Khoze, and M. P. Mattis, "On $\mathcal{N}=2$ supersymmetric QCD with 4 flavors," Nucl. Phys. B492 (1997) 607-622, arXiv:hep-th/9611016.

[10] T. W. Grimm, A. Klemm, M. Mariño, and M. Weiss, "Direct integration of the topological string," JHEP 08 (2007) 058, arXiv:hep-th/0702187.

[11] T. J. Hollowood, A. Iqbal, and C. Vafa, "Matrix Models, Geometric Engineering and Elliptic Genera," JHEP 03 (2008) 069, arXiv:hep-th/0310272.

[12] A. Iqbal, C. Kozçaz, and C. Vafa, "The refined topological vertex," arXiv:hep-th/0701156.

[13] B. Ponsot and J. Teschner, "Liouville bootstrap via harmonic analysis on a noncompact quantum group," arXiv:hep-th/9911110.

[14] J. Teschner, "A lecture on the Liouville vertex operators," Int. J. Mod. Phys. A19S2 (2004) 436-458, arXiv:hep-th/0303150.

[15] J. Teschner, "From Liouville theory to the quantum geometry of Riemann surfaces," arXiv:hep-th/0308031.

[16] J. Teschner, "An analog of a modular functor from quantized Teichmuller theory," arXiv:math/0510174.

[17] J. Teschner, "Nonrational conformal field theory," arXiv:0803.0919 [hep-th].

[18] L. Hadasz, Z. Jaskólski, and P. Suchanek, "Modular Bootstrap in Liouville Field Theory," arXiv:0911.4296 [hep-th].

[19] D. V. Nanopoulos and D. Xie, "On Crossing Symmmetry and Modular Invariance in Conformal Field Theory and S Duality in Gauge Theory," Phys. Rev. D80 (2009) 105015, arXiv:0908.4409 [hep-th].

[20] T. Eguchi and H. Kanno, "Geometric transitions, Chern-Simons gauge theory and Veneziano type amplitudes," Phys. Lett. B585 (2004) 163-172, arXiv:hep-th/0312234.

[21] A. Iqbal and A.-K. Kashani-Poor, "The vertex on a strip," Adv. Theor. Math. Phys. 10 (2006) 317-343, arXiv:hep-th/0410174.

[22] E. Witten, "Quantum background independence in string theory," arXiv:hep-th/9306122. 
[23] M. Aganagic, A. Klemm, M. Mariño, and C. Vafa, "The topological vertex," Commun. Math. Phys. 254 (2005) 425-478, arXiv:hep-th/0305132.

[24] M. Aganagic, R. Dijkgraaf, A. Klemm, M. Mariño, and C. Vafa, "Topological strings and integrable hierarchies," Commun. Math. Phys. 261 (2006) 451-516, arXiv:hep-th/0312085.

[25] H. Ooguri, A. Strominger, and C. Vafa, "Black hole attractors and the topological string," Phys. Rev. D70 (2004) 106007, arXiv: hep-th/0405146.

[26] M. Mariño and N. Wyllard, "A note on instanton counting for $\mathcal{N}=2$ gauge theories with classical gauge groups," JHEP 05 (2004) 021, arXiv:hep-th/0404125.

[27] N. Nekrasov and S. Shadchin, "ABCD of instantons," Commun. Math. Phys. 252 (2004) 359-391, arXiv:hep-th/0404225.

[28] S. Shadchin, "Cubic curves from instanton counting," JHEP 03 (2006) 046, arXiv:hep-th/0511132.

[29] J. Teschner, "Liouville theory revisited," Class. Quant. Grav. 18 (2001) R153-R222, arXiv:hep-th/0104158.

[30] Y. Nakayama, "Liouville field theory: A decade after the revolution," Int. J. Mod. Phys. A19 (2004) 2771-2930, arXiv: hep-th/0402009.

[31] A. A. Belavin, A. M. Polyakov, and A. B. Zamolodchikov, "Infinite conformal symmetry in two-dimensional quantum field theory," Nucl. Phys. B241 (1984) $333-380$.

[32] H. Sonoda, "Sewing Conformal Field Theories," Nucl. Phys. B311 (1988) 401.

[33] G. W. Moore and N. Seiberg, "Classical and Quantum Conformal Field Theory," Commun. Math. Phys. 123 (1989) 177.

[34] P. Di Francesco, P. Mathieu, and D. Sénéchal, Conformal field theory. Springer, 1997.

[35] E. W. Barnes, "The Theory of the Double Gamma Function," Phil. Trans. Roy. Soc. Lond. A 196 (1901) 265-387, JSTORE:90809.

[36] F. Fucito, J. F. Morales, and R. Poghossian, "Instantons on quivers and orientifolds," JHEP 10 (2004) 037, arXiv:hep-th/0408090. 